\title{
Evaluation of multivariate GARCH models in an optimal asset allocation framework
}

\author{
Nor Syahilla Abdul Aziz*, Spyridon Vrontos, Haslifah M. Hasim \\ Department of Mathematical Sciences, University of Essex, Colchester, CO4 3SQ Essex, United Kingdom
}

\section{A R T I C L E I N F O}

\section{Keywords:}

Asset management

Asset allocation

GARCH

Copula

Dynamic conditional correlation

Portfolio optimisation

JEL classification:

C58

G11

G15

G17

G32

\begin{abstract}
A B S T R A C T
This paper analyses plethora of advanced multivariate econometric models, which forecast the mean and variance-covariance of the asset returns to create optimal asset allocation models. Most existing studies use a limited number of Generalized Autoregressive Conditional Heteroscedasticity (GARCH) models. In this study, we provide an in-depth knowledge of large asset modeling and optimization strategies for solving a portfolio selection problem involving the dynamic conditional correlation models (DCC). Specifically, we use symmetric GARCH models and an asymmetric version of it (GJR-GARCH). Several studies have also tried to examine the effectiveness of using parametric copula in estimating portfolio risk measures but their results have been inconclusive. We are interested in evaluating if Copula-GARCH could be an optimal model for assessing the performance of a portfolio. This study, therefore, implemented various Copula-GARCH based models using the static and dynamic estimation of the correlation. By employing different model specifications, we are able to explore the empirical applicability of the multivariate GARCH models when estimating large conditional covariance matrices. In constructing the optimal portfolios, we evaluate the minimum variance, mean-variance, maximising Sharpe ratio, mean-CVaR, and maximization of Sortino ratio. We compare the out-of-sample performance for each of the models based on the risk-adjusted performance for a portfolio with and without short sales, consisting eight stocks and four bond indices of 10 years maturity, in the United States (US), United Kingdom (UK), Germany, Japan, Netherlands, Canada and Hong Kong. Our results suggest that the dynamic models are more capable of delivering better performance gains than the static models. These models reduce portfolio risk and improve the realized return in the out-of-sample period. This paper concludes that by adding copula functions to the model, it does not give a better performance model when compared to the dynamic correlation model.
\end{abstract}

\section{Introduction}

With today's challenging environments and highly volatile markets, the study of asset management has become important for organizations in managing their assets to obtain the best possible returns. Asset allocation aims to balance the risk and return by adjusting the percentage of each asset in an investment portfolio to meet the investor's goals, investment objectives, risk tolerance, and investment horizon.

The future forecast is highly dependent on the choice of the volatility modeling. It is known that volatility is not directly

\footnotetext{
* Corresponding author.

E-mail address: nsbabd@essex.ac.uk (N.S. Abdul Aziz).
} 
observable, which makes it important to have a good model to predict future volatilities. Obtaining an optimal portfolio requires estimating and forecasting very large conditional covariance matrices of the asset returns which depend on many parameters (Billio, Caporin, \& Gobbo, 2006; Lee, Chiou, \& Lin, 2006). Over the past years, several studies have developed methods and approaches to examine the dynamics of covariance of assets. Previous studies on asset allocation, focus on a limited or specific econometric model to model its asset return covariances (see, for example, Koivu, Pennanen, \& Koivu (2005), Ferstl \& Weissensteiner (2011), Hoevenaars, Molenaar, Schotman, \& Steenkamp (2013)). One of the widely used model is the Vector Autoregressive (VAR) model which is an extension of the univariate autoregressive model to dynamic multivariate time series.

To deal with a large number of parameters in multivariate models, Bollerslev, Engle, and Wooldridge (1998) suggest a Constant Conditional Correlation (CCC) model such that the conditional correlations are assumed to be constant. This model reduces the number of parameters and thus simplifies the estimations considerably. However, the assumption of this model may not be realistic because the conditional shocks are correlated only in the same market, and not across markets (Chang, McAleer, \& Tansuchat, 2013). Engle (2002) proposes a generalization of CCC model, by allowing the correlations to change over time, known as the Dynamic Conditional Correlation (DCC) model. This model is estimated using a two-steps approach: the estimation of mean and variance by a series of univariate GARCH models, and the correlation estimation.

Alternative DCC models also have been proposed in the literature which are aiming to solve problems associated with the basic DCC model. The limits of the DCC model are constrained by the equal dynamics for the correlations of all the assets (Billio et al., 2006). To avoid this problem, Billio et al. (2006) propose the Flexible DCC (FDCC) model such that the correlation dynamic is constrained to be equal only between $w$ groups of variables, providing flexible dynamics. Another study by Otranto (2010) examines the performance of optimal asset allocation strategies using FDCC models with regime switching as compared to alternative models. Recently, Aielli (2013) suggests a more tractable dynamic conditional correlation model, known as a corrected DCC model or cDCC model, which involves the three-step approach that is feasible with large systems and provides unbiased estimations (Boudt et al., 2013). Recent proposals of multivariate GARCH models include the asymmetric DCC model (aDCC) of Cappiello, Engle, and Sheppard (2006), the Copula GARCH of Patton (2004), the dynamic equicorrelation (DECO) model of Engle and Kelly (2012) and the smooth transition conditional correlation (STCC-GARCH) of Silvennoinen and Terasvirta (2015).

The copula theory was introduced by Sklar (1959). It states that any multivariate distribution function can be decomposed into its marginal distributions and a copula function. The application of copula function was only being introduced in the late 1990's in actuarial science and finance by Embrechts, McNeil, and Straumann (1999) and Li (2000), and more recently by Hurlimann (2014) and $\mathrm{Wu}$ and Lin (2014). Over the years, copula function has been popular in the financial research, especially because of its application to risk management and asset allocation. However, there are very few studies assessing the out-of-sample performance of a portfolio based on the copula model (see, for example, Patton (2004), Ricetti (2013), Wu \& Lin (2014), Kresta (2015)).

This paper makes several contributions to the literature. First, this paper involves a large class of different advanced multivariate DCC GARCH models. We use 26 different model specifications using multivariate GARCH processes in modeling the mean returns and variance-covariance matrices. Specifically, we use a symmetric GARCH model and an asymmetric version of it (GJR-GARCH), such that the models are implemented with the multivariate normal and student distributions. For the conditional mean dynamics, this study allows a constant, univariate autoregressive (AR), autoregressive-moving average (ARMA) or vector autoregression (VAR) model to be fit. In general, the model specifications to model covariances includes DCC models, aDCC models, FDCC models, Generalized Orthogonal GARCH (GO-GARCH) and copula-GARCH models. For the normal DCC models, the conditional mean is jointly estimated with the first stage GARCH while, whereas for the GO-GARCH models, the dynamics for the conditional mean are defined from the general ARFIMAX model of Engle, Lilien, and Robins (1987), assuming constant variance to obtain the parameter estimates. The copula-GARCH models are implemented using the static and dynamic estimation of the correlation. We apply different model specifications to obtain out-of-sample forecasts of the mean returns and the conditional covariance matrix of all assets and use the estimated matrices to construct optimal short selling-constrained and unconstrained portfolios. The portfolio is constructed by using different optimization strategies such as minimum variance, mean-variance, maximization of the Sharpe ratio, maximization of Sortino ratio for a given risk and mean-CVaR. To our best knowledge, we are the only one who provide a comprehensive comparative study involving various multivariate GARCH models in an optimal asset allocation setting. From this study, we can capture different characteristics in the data which improve the portfolio performance and are useful for the construction of optimal portfolios. This paper differs from previous studies by assessing the out-of-sample performance of large groups of different multivariate DCC GARCH models in different optimization schemes considering portfolios with and without short sales. Most of the existing literature compares the performance of limited GARCH models, and they are based on a specific optimization model. Several studies have tried to examine the effectiveness of using parametric copula in estimating portfolio risk measures, but they have been inconclusive. It is unclear whether it is optimal to use copula-GARCH over the sophisticated DCC model. This study, therefore, will provide useful insights for those wishing to explore the different GARCH models that are available, from the simplest autoregressive models up to more complex models. and use a dynamic approach for asset allocation and portfolio construction purposes.

The remainder of the paper is organized as follows: Section 2 discusses the econometric models we use to model the asset returns covariances. Section 3 describes the portfolio optimization strategies used to construct optimal asset portfolios and look at how the models evolve. Section 4 presents the data, empirical analysis and results of the proposed models and methods, while Section 5 provides concluding remarks and identifies the shortcomings and future implications of the study.

\section{Econometric models for asset return}

The multivariate GARCH requires mean and covariance estimation values as inputs for the portfolio optimisation. In this paper, 
we consider different econometric methodologies to model mean asset return and covariances.

Volatility has some characteristics that are not directly noticeable but are commonly seen in asset returns; i.e, the presence of volatility clustering in the data, a volatility jump is rare, stationary, and has a leverage effect (Tsay, 2013). Various volatility models were introduced, particularly to correct the weaknesses of their inability to capture the characteristics mentioned previously.

Consider the vector stochastic process $x_{t}$ which is the $T \times 1$ vector of log returns of $n$ assets at time $t$ and $\mu_{t}$ is the $T \times 1$ vector of the expected value of conditional $x_{t}$ such that $\mu_{t}$ may be modeled as a constant vector or a time series model. The multivariate GARCH models are written as

$$
x_{t}=\mu_{t}+\epsilon_{t}, \quad \epsilon_{t} \mid \Phi_{t-1} \sim N\left(0, H_{t}\right),
$$

where $\epsilon_{t}$ is the residuals of the process which follows a conditionally multivariate normal distribution with mean 0 and time-varying conditional covariance matrix $H_{t}$, given $\Phi_{t-1}$ the information set at time $t-1$. The residuals are modelled as

$$
\epsilon_{t}=H_{t}^{\frac{1}{2}} z_{t}
$$

where $H_{t}$ is $T \times n$ positive definite matrix of conditional variances of $x_{t}$ at time $t . H_{t}^{\frac{1}{2}}$ is the Cholesky factorization of the time varying conditional covariance matrix of $H_{t}$. The symbol $z_{t}$ is $T \times 1$ vector of independent and identically distributed random errors such that $E\left[z_{t}\right]=0$ and $E\left[z_{t} z_{t}^{\prime}\right]=I_{T}$, whereby $I_{T}$ denotes the identity matrix of order $T$. The conditional covariance matrix $H_{t}$ of $x_{t}$ may be defined as

$$
\begin{aligned}
\operatorname{Var}\left[x_{t} \mid \tilde{\mathscr{F}}_{t-1}\right]=\operatorname{Var}_{t-1}\left[x_{t}\right. & =\operatorname{Var}_{t-1}\left[\epsilon_{t}\right] \\
& =H_{t}^{\frac{1}{2}} \operatorname{Var}_{t-1}\left(\epsilon_{t}\right)\left(H_{t}^{\frac{1}{2}}\right)^{\prime}=H_{t},
\end{aligned}
$$

where $\mathscr{F}_{t}=\left\{x_{t}, x_{t-1}, \ldots\right\}$. We use different specifications for multivariate GARCH processes of forecasting mean asset return and covariances. By using different econometric models, we are able to capture different characteristics in the data which improve the portfolio performance and are useful for the construction of optimal portfolios. We then compare the results of each model to see their ability in optimizing portfolio.

\subsection{Modeling mean returns}

There are various models for time series which are divided into the AR and MA models. The AR(m) model can be written as

$$
x_{t}=\sum_{i=1}^{m} \phi_{i} x_{t-i}+\epsilon_{t} .
$$

For the moving average, MA (n) refers to

$$
x_{t}=\epsilon_{t}-\sum_{j=1}^{n} \theta_{j} \epsilon_{t-j} .
$$

These models are commonly used to generate new models, i.e., ARMA or VAR models. We model the mean returns using different estimation processes, either using a constant mean, AR, ARMA or VAR models.

The ARMA model provides a parsimonious parametrization and further simplification in modeling multivariate time series. It has both stationary stochastic processes of the autoregression and moving average methods, which are applied to a multivariate time series data. The ARMA $(m, n)$ model refers to the $m$ autoregressive terms and $n$ moving average terms, which includes the AR ( $m$ ) and MA $(n)$ models. Thus, the mean of the process modeled by ARMA $(m, n)$ is

$$
x_{t}=\phi_{0}+\sum_{i=1}^{m} \phi_{i} x_{t-i}+\sum_{j=1}^{n} \theta_{j} \epsilon_{t-j}+\epsilon_{t},
$$

where $\phi_{0} \in \mathbb{R}$ and $\alpha_{i}$ and $\beta_{i}$ are $T \times n$ diagonal matrices. The autoregressive coefficients is denoted by $\phi_{i}$ and moving average coefficients is denoted by $\theta_{j}$ if there exist real coefficients $\phi_{1}, \ldots, \phi_{m}$ and $\theta_{1}, \ldots, \theta_{n}$, such that $\epsilon_{t}$ is the linear innovation process of $x_{t}$. $\phi_{i}$ and $\theta_{j}$ are $T \times n$ matrices with $\phi_{i} \neq 0$ and $\theta_{j} \neq 0$.

The VAR model is one of the most commonly used multivariate econometric models. We allow the conditional mean to follow a VAR structure, where the model can be represented by

$$
x_{t}=c+\sum_{i=1}^{m} \phi_{i} x_{t-i}+\epsilon_{t},
$$

where $x_{t}$ is vectors and $c$ denotes $T \times 1$ dimensional vector of constants. $\phi_{1}, \ldots, \phi_{m}$ is parameter matrices. This model has an important characteristic which is its stability. It generates stationary time series with time invariant means, variances and covariances.

\subsection{Modeling covariances matrix}

We use different specifications for multivariate GARCH processes of forecasting a variance-covariances matrix, i.e., (i) Dynamic 
Conditional Correlation (DCC), (ii) Asymmetric Dynamic Conditional Correlation (aDCC), (iii) Flexible Dynamic Condition Correlation (FDCC), (iv) Generalized Orthogonal GARCH (GOGARCH), and (v) Copula GARCH (C-GARCH).

\subsubsection{Dynamic Conditional Correlation (DCC)}

The DCC model was proposed by Engle (2002), which is a generalization of the Constant Conditional Correlation (CCC) model from Bollerslev et al. (1998), to allow for the time-varying correlation matrix of multiple asset returns. In the CCC model, the conditional covariance matrix is decomposed into conditional standard deviations and a constant correlation as

$$
H_{t}=D_{t} R D_{t} \text {, }
$$

where $D_{t}$ is the $T \times n$ diagonal matrix of time-varying standard deviations from univariate GARCH models, $\left\{D_{t}=\operatorname{diag}\left(\sqrt{h_{1 t}}, \ldots, \sqrt{h_{n t}}\right)\right.$, and $R=\rho_{i j}$ is the positive definite constant conditional correlation matrix with $\rho_{i i}=1$ for $i=1, \ldots, n$. The off-diagonal elements of $H_{t}$, are given by

$$
\left[H_{t}\right]_{i j}=\rho_{i j} \sqrt{h_{i t}} \sqrt{h_{j t}}, \quad i \neq j .
$$

This model is computationally attractive and simple because of the constant correlation. However, the assumptions of constant conditional correlations may be unrealistic in practice and it may be too restrictive in some cases. The DCC model allows the timevarying correlation dynamics, $R=R_{t}$. It is defined as

$$
\begin{aligned}
x_{t} & =\mu_{t}+\epsilon_{t} \\
\epsilon_{t} & =H_{t}^{\frac{1}{2}} z_{t} \\
H_{t} & =D_{t} R_{t} D_{t},
\end{aligned}
$$

such that $x_{t}$ is a $T \times 1$ vector of $\log$ returns of $n$ assets at time $t . \epsilon_{t}$ is a $T \times 1$ vector of mean corrected returns of $n$ assets at time $t$ such that $E\left[\epsilon_{t}\right]=0$ and $\operatorname{Cov}\left[\epsilon_{t}\right]=H_{t} . \mu_{t}$ is a $T \times 1$ vector of the expected value of the conditional $x_{t} . H_{t}$ is a $T \times n$ matrix of conditional variances of $\epsilon_{t}$ at time $t$, and $D_{t}$ is a $T \times n$ diagonal matrix of conditional standard deviation of $\epsilon_{t}$ at time $t$. Note that the conditional correlation matrix of $\epsilon_{t}$ is now time varying and denoted by a symbol $R_{t}$. While $z_{t}$ is $T \times 1$ vector of independent and identically distributed random errors such that $E\left[z_{t}\right]=0$ and $E\left[z_{t} z_{t}^{\prime}\right]=I_{T}$. The conditional variances, $H_{t}$ can be estimated separately by a simple univariate GARCH specification of

$$
H_{t}=\sigma_{i, t}^{2}=g_{i}+\sum_{l=1}^{q} \beta_{i} \sigma_{i, t-l}^{2}+\sum_{m=1}^{p} \alpha_{i} \epsilon_{i, t-m}^{(2)},
$$

where $g_{i}$ is $T \times 1$ vector of constant, $\alpha_{i}$ and $\beta_{i}$ are $T \times n$ diagonal matrices. $\epsilon_{t-m}^{(2)}=\epsilon_{t-m} \odot \epsilon_{t-m}$ is the Hadamard product, which is the element by element product. $H_{t}$ is a positive definite matrix such that $g_{i}>0$ and $\alpha_{i}, \beta_{i} \geqslant 0 . R_{t}$ is also a positive definite conditional correlation matrix of the standardized disturbances of $z_{t}$ such that

$$
z_{t}=D_{t}^{-1} \epsilon_{t} \sim N\left(0, R_{t}\right)
$$

Thus, the elements of $H_{t}=D_{t} R_{t} D_{t}$ with $\rho_{i i}=1$ can be written as

$$
\left[H_{t}\right]_{i j}=\rho_{i j, t} \sqrt{h_{i t} h_{j t}} .
$$

The conditions for the positivity of the covariance matrix $H_{t}$ requires $R_{t}$ to be positive definite, $g_{i}$ and all diagonal elements of matrices $\beta_{i}$ and $\alpha_{i}$ are all positive. Therefore, we need to decompose $R_{t}$ into:

$$
\begin{aligned}
R_{t} & =\left(Q_{t}^{*}\right)^{-1} Q_{t}\left(Q_{t}^{*}\right)^{-1} \\
Q_{t} & =(1-a-b) \bar{Q}+a z_{t-1} z_{t-1}^{\prime}+b Q_{t-1} .
\end{aligned}
$$

$\bar{Q}=\operatorname{Cov}\left[z_{t} z_{t}^{\prime}\right]=E\left[z_{t} z_{t}^{\prime}\right] \quad$ is a $T \times n$ unconditional matrix of the standardised errors $z_{t}$, where $z_{t}=D_{t}^{-1} \epsilon_{t}$. $Q_{t}^{*}=\operatorname{diag}\left(\sqrt{q_{1 t}}, \sqrt{q_{2 t}}, \ldots, \sqrt{q_{n t}}\right) ; a$ and $b$ are non-negative parameters to be estimated such that $a+b<1$ to ensure stationarity and positive definiteness of $Q_{t}$. As in Engle (2002), $\bar{Q}_{t}$ can be estimated by

$$
\bar{Q}_{t}=\frac{1}{T} \sum_{t=1}^{T} z_{t} z_{t}^{\prime}
$$

In general, as in Engle (2002), the DCC-GARCH model is given by

$$
Q_{t}=\left(1-\sum_{m=1}^{M} a_{m}-\sum_{n=1}^{N} b_{n}\right) \overline{Q_{t}}+\sum_{m=1}^{M} a_{m} z_{t-1} z_{t-1}^{\prime}+\sum_{n=1}^{N} b_{n} Q_{t-1} .
$$

This model is estimated using a two-step approach: the first implies the estimation of univariate GARCH, and the second step is the correlation estimation. The number of the estimated parameters in the correlation process of DCC-GARCH is independent of the number of series correlated, hence allowing for a potentially large correlation matrices to be feasibly estimated. The limitation of this model is to hypothesis the same correlation dynamics of all the assets (Billio et al., 2006; Otranto, 2010). 


\subsubsection{Asymmetric Dynamic Conditional Correlation (aDCC)}

Cappiello et al. (2006) introduced an aDCC model to investigate whether conditional variances, covariances, and correlations of assets in a portfolio are sensitive to the sign of past innovations. Compared to the DCC model, this model further explores whether the positive and negative shocks are of the same magnitude or have different impacts. As in Eq. (10), the matrix $H_{t}$ is decompose into $H_{t}=D_{t} R_{t} D_{t}$.

The conditional variances, $H_{t}$ is assumed to follow the GJR-GARCH models,

$$
\sigma_{i, t}^{2}=g_{i}+\sum_{l=1}^{q} \beta_{i} \sigma_{i, t-l}^{2}+\sum_{m=1}^{p}\left(\alpha_{i}+\gamma_{i} \Psi_{i, t-m}\right) \epsilon_{i, t-m}^{(2)},
$$

where $w_{i}, \alpha_{i}, \beta_{i}, \gamma_{i}>0$, and $\Psi_{i, t-m}$ is an indicator function such that $\Psi_{i, t-m}=1$, if $\epsilon_{i, t}<0$ and 0 otherwise. In this model, Eq. (14) can be extended to consider the asymmetries, which can be written as

$$
Q_{t}=\left(\bar{Q}-A^{\prime} \bar{Q} A-B^{\prime} \bar{Q} B-G^{\prime} \bar{N} G\right)+A^{\prime} z_{t-1} z_{t-1}^{\prime} A+B^{\prime} Q_{t-1} B+G^{\prime} z_{t} z_{t}^{\prime} G
$$

where $\bar{N}=E\left[z_{t} z_{t}^{\prime}\right]$ and $n_{t}=\Psi\left[z_{t-1}<0\right] \odot z_{t}$. The $\odot$ symbol is the element by element product of the residuals if shocks are negative, and $n_{t}=0$, otherwise. The asymmetric term is denoted by a symbol $G$, which captures the period when both markets experience negative shocks, resulting $\left[n n_{t}^{\prime}\right]=\Psi_{t}$. The symbols $A, B$, and $G$ represent the diagonal parameter matrices, where $A, B, G>0$ to ensure the positiveness and stationarity of $Q_{t} \cdot \bar{Q}=E\left[z_{t} z_{t}^{\prime}\right]$ is a $T \times n$ unconditional matrix of the standardised errors $z_{t}$.

\subsubsection{Flexible Dynamic Condition Correlation (FDCC)}

Billio et al. (2006) proposed the FDCC model that allows for equal correlation dynamics between $w$ groups of assets, providing a flexible parameterisation of correlation dynamics. The FDCC model, which is the extension of the DCC model, introduces a block diagonal structure to solve the problem of equal correlations dynamics in the assets. As in Billio et al. (2006), this model can parsimoniously be written as

$$
\begin{aligned}
& x_{t}=\mu_{t}+\epsilon_{t} \\
& H_{t}=D_{t} R_{t} D_{t} \\
& R_{t}=\left(Q_{t}^{*}\right)^{-1} Q_{t}\left(Q_{t}^{*}\right)^{-1} \\
& Q_{t}=c c^{\prime}+a a^{\prime} \odot \eta_{t} \eta_{t}^{\prime}+b b^{\prime} \odot Q_{t-1},
\end{aligned}
$$

where $\eta_{t}$ is the standardised residuals, $\eta_{t}=D_{t}^{-1} \epsilon_{t}$ and the variables $c, a$ and $b$ are partitioned $n$-dimensional vectors of groups of assets,

$$
a=\left[a_{1} \times i_{m_{1}}^{\prime}, a_{2} \times i_{m_{2}}^{\prime}, \ldots, a_{k} \times i_{m_{k}}^{\prime}\right]^{\prime}
$$

where $m_{i}(i=1, \ldots, k)$ is the number of assets in the group $i$; and similarly for $b$ and $c$. $i_{h}$ is an $h$-dimensional vector of ones. The coefficients must satisfy these constraints: $a_{i} a_{j}+b_{i} b_{j}<1(i, j=1, \ldots, k)$, such that $k$ is the number of blocks or asset classes. In this study, we divided the assets into two blocks: stock and bond indices groups. This is reasonable since the correlation dynamics within the stock group is almost similar from one asset to another, and this is also the same for bond indices.

\subsubsection{Generalised orthogonal GARCH (GO-GARCH)}

OGARCH was introduced by Ding (1994) and Alexander and Chibumba (1996). The observed time series can be linearly transformed to a set of uncorrelated time series using a principal component analysis. This model has commonly been used in much research to model the conditional covariance of financial time series due to its feasibility in estimating large covariance matrices (Weide, 2002; Luo, Seco, \& Wu, 2015). For non-Gaussian data, the independent component analysis (ICA) is used to perform the orthogonal transformation. Weide (2002) applies the concept of ICA to propose the generalized OGARCH model for volatility modeling. It consists of a set of conditionally uncorrelated univariate GARCH and a linear map that allows the linkage between these components and the observed data (Boswijk et al., 2006).

Consider a set of $n$ assets with returns $x_{t}$, which are observed for $T$ periods, with conditional mean of $E\left[x_{t} \mid \mathscr{F}_{t-1}\right]=m_{t} . \mathscr{F}_{t}$ is the information set at time $t$, which is the $\sigma$-algebra generated by the lagged values of the outcome process of $x_{t}$, i.e, $\mathscr{F}_{t}=\sigma\left(x_{t-1}, x_{t-2}, \ldots\right)$. The GO-GARCH model of Weide (2002) maps $x_{t}-m_{t}$ onto a set of unobserved independent factors $f_{t}$. The process $x_{t}$ satisfies the representation

$$
\begin{aligned}
& x_{t}=m_{t}+\epsilon_{t} \\
& \epsilon_{t}=C f_{t},
\end{aligned}
$$

where $f_{t}=\left(f_{1 t}, \ldots, f_{n t}\right)^{\prime} . C$ is a non singular matrix which is invertible and constant over time. It may be decomposed into the dewhitening matrix $\Sigma_{t}^{1 / 2}$, i.e., the square root of unconditional covariance and an orthogonal matrix $U_{0}$. $U_{0}$ can be estimated using a computational method for separating multivariate mixed signals into additive statistically independent and non-Gaussian components using ICA (Broda \& Paolella, 2009; Zhang \& Chan, 2009).

Let $C$ be the map that links the uncorrelated components with the unobserved process, so that

$$
C=\Sigma_{t}^{1 / 2} U_{0}
$$



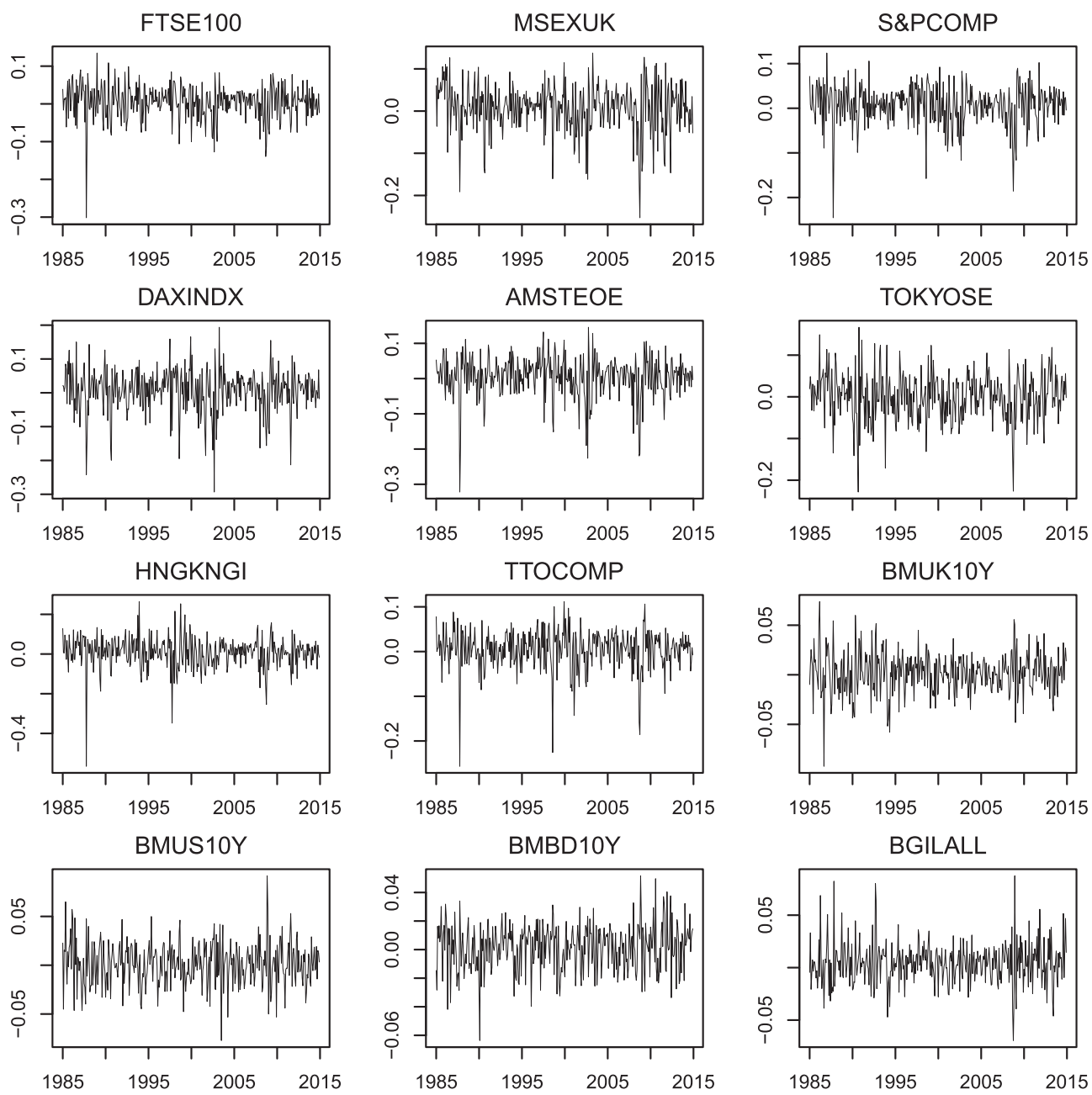

Fig. 1. Plots of the monthly analysed asset returns series from January 1985 to December 2014.

Table 1

Statistical characteristics and Ljung-Box of historical monthly returns for the analysed assets from January 1985 to December 2014 . The Ljung-box test is computed using 12 lags.

\begin{tabular}{|c|c|c|c|c|c|c|c|}
\hline \multirow[t]{2}{*}{ Assets } & \multicolumn{5}{|c|}{ Rate of Returns, $R_{t}$} & \multirow{2}{*}{$\begin{array}{c}\text { Sq. returns, } R_{t}^{2} \\
\text { Q(12) }\end{array}$} & \multirow{2}{*}{$\begin{array}{c}\text { Abs. returns, }\left|R_{t}\right| \\
\mathrm{Q}(12)\end{array}$} \\
\hline & Mean & Stdev & Skewness & Kurtosis & $\mathrm{Q}(12)$ & & \\
\hline FTSE100 & 0.0046 & 0.0455 & -1.1387 & 5.3324 & 7.1110 & 8.1196 & 34.587 \\
\hline MSEXUK & 0.0073 & 0.0563 & -0.8630 & 1.8616 & 14.1310 & $55.498^{* *}$ & $72.312^{* *}$ \\
\hline S.PCOMP & 0.0070 & 0.0444 & -1.1008 & 3.6350 & 7.7062 & 20.4750 & $51.275^{* *}$ \\
\hline DAXINDX & 0.0069 & 0.0631 & -0.9101 & 2.6948 & 7.3127 & $23.453^{* *}$ & $35.758^{* *}$ \\
\hline AMSTEOE & 0.0044 & 0.0581 & -1.3154 & 4.2702 & 15.0920 & $43.91^{* *}$ & $57.092^{* *}$ \\
\hline TOKYOSE & 0.0012 & 0.0564 & -0.3667 & 1.2278 & 16.2010 & $30.392^{* *}$ & 11.320 \\
\hline HNGKNGI & 0.0083 & 0.0782 & -1.3354 & 8.8759 & $22.723^{* *}$ & 3.5307 & $31.804^{* *}$ \\
\hline TTOCOMP & 0.0050 & 0.0437 & -1.4879 & 6.1225 & 15.1270 & 7.1863 & $30.113^{* *}$ \\
\hline BMUK10Y & 0.0021 & 0.0206 & -0.1586 & 1.3692 & 19.9310 & $40.983^{* *}$ & $37.633^{* *}$ \\
\hline BMUS10Y & 0.0015 & 0.0218 & 0.0523 & 0.9303 & 16.3820 & 15.2260 & 9.5042 \\
\hline BMBD10Y & 0.0015 & 0.0162 & -0.2619 & 0.4050 & $27.532^{* *}$ & 8.5521 & 11.2980 \\
\hline BGILALL & 0.0044 & 0.0196 & 0.5009 & 2.4422 & 15.7770 & $23.109^{* *}$ & 19.5850 \\
\hline
\end{tabular}

Note: ${ }^{* *}$ represents the $p$-value of $<0.05$, indicating that the null hypothesis of no autocorrelation is rejected at $95 \%$ confidence level (critical value of 21.026). 
Table 2

Correlations for the analysed asset returns series from January 1985 to December 2014.

\begin{tabular}{|c|c|c|c|c|c|c|c|c|c|c|c|c|}
\hline Assets & FTSE100 & MSEXUK & S\&PCOMP & DAXINDX & AMSTEOE & TOKYOSE & HNGKNGI & ТTOСОМР & BMUK10Y & BMUS10Y & BMBD10Y & BGILALL \\
\hline FTSE100 & 1.0000 & 0.6990 & 0.7950 & 0.6840 & 0.7560 & 0.4300 & 0.6300 & 0.6850 & 0.1480 & 0.0736 & 0.0709 & 0.2200 \\
\hline MSEXUK & - & 1.0000 & 0.7360 & 0.7830 & 0.7270 & 0.4920 & 0.5770 & 0.6530 & 0.0008 & 0.0586 & 0.0772 & 0.1450 \\
\hline S\&PCOMP & - & - & 1.0000 & 0.6910 & 0.7320 & 0.4630 & 0.6100 & 0.7820 & 0.0080 & 0.0259 & 0.1140 & 0.1410 \\
\hline DAXINDX & - & - & - & 1.0000 & 0.8140 & 0.4220 & 0.5240 & 0.5970 & 0.0499 & 0.1820 & 0.0997 & 0.0579 \\
\hline AMSTEOE & - & - & - & - & 1.0000 & 0.4430 & 0.5680 & 0.6660 & 0.0806 & 0.2210 & 0.1580 & 0.0808 \\
\hline TOKYOSE & - & - & - & - & - & 1.0000 & 0.3570 & 0.4480 & 0.0409 & 0.1120 & 0.0836 & 0.0668 \\
\hline HNGKNGI & - & - & - & - & - & - & 1.0000 & 0.6500 & 0.0210 & 0.0918 & 0.1180 & 0.1040 \\
\hline TTOCOMP & - & - & - & - & - & - & - & 1.0000 & 0.0375 & 0.0776 & 0.1290 & 0.1590 \\
\hline BMUK10Y & - & - & - & - & - & - & - & - & 1.0000 & 0.5820 & 0.7090 & 0.5820 \\
\hline BMUS10Y & - & - & - & - & - & - & - & - & - & 1.0000 & 0.6930 & 0.3010 \\
\hline BMBD10Y & - & - & - & - & - & - & - & - & - & - & 1.0000 & 0.3920 \\
\hline BGILALL & - & - & - & - & - & - & - & - & - & - & - & 1.0000 \\
\hline
\end{tabular}

Table 3

Descriptive statistics and out-of-sample performance of minimum variance efficient portfolio without short sale for the econometric models under study from January 2005 to December 2014.

\begin{tabular}{|c|c|c|c|c|c|c|c|}
\hline Model & Return & Cumulative Return & Risk & Sharpe Ratio & VaR@90\% & VaR@95\% & VaR@99\% \\
\hline DCC-MVN & 0.0027 & 0.3272 & 0.0191 & 0.1447 & -0.0141 & -0.0245 & -0.0508 \\
\hline DCC-MVT & 0.0031 & 0.3732 & 0.0191 & 0.1643 & -0.0159 & -0.0215 & -0.0625 \\
\hline aDCC-MVN & 0.0033 & 0.4007 & 0.0192 & 0.1723 & -0.0137 & -0.0238 & -0.0627 \\
\hline aDCC-MVT & 0.0038 & 0.4548 & 0.0192 & 0.2013 & -0.0151 & -0.0239 & -0.0663 \\
\hline FDCC-MVN & 0.0027 & 0.3223 & 0.0192 & 0.1420 & -0.0142 & -0.0244 & -0.0507 \\
\hline VAR-MVN & 0.0026 & 0.3081 & 0.0191 & 0.1329 & -0.0136 & -0.0246 & -0.0681 \\
\hline VAR-MVT & 0.0023 & 0.2780 & 0.0188 & 0.1207 & -0.0141 & -0.0252 & -0.0611 \\
\hline ARMA-MVN & 0.0027 & 0.3228 & 0.0194 & 0.1430 & -0.0140 & -0.0229 & -0.0513 \\
\hline ARMA-MVT & 0.0027 & 0.3286 & 0.0188 & 0.1452 & -0.0138 & -0.0235 & -0.0673 \\
\hline GG-MVN & 0.0031 & 0.3665 & 0.0188 & 0.1653 & -0.0155 & -0.0255 & -0.0652 \\
\hline ARMA-GG-MVN & 0.0026 & 0.3119 & 0.0188 & 0.1371 & -0.0165 & -0.0275 & -0.0656 \\
\hline VAR-GG-MVN & 0.0026 & 0.3104 & 0.0184 & 0.1428 & -0.0160 & -0.0299 & -0.0612 \\
\hline SCop-MVN & 0.0022 & 0.2696 & 0.0191 & 0.1209 & -0.0195 & -0.0331 & -0.0605 \\
\hline SCop-MVT & 0.0021 & 0.2526 & 0.0201 & 0.1012 & -0.0212 & -0.0320 & -0.0663 \\
\hline ARMA-SCop-MVN & 0.0020 & 0.2388 & 0.0189 & 0.1060 & -0.0191 & -0.0315 & -0.0656 \\
\hline ARMA-SCop-MVT & 0.0021 & 0.2482 & 0.0187 & 0.1109 & -0.0187 & -0.0285 & -0.0689 \\
\hline VAR-SCop-MVN & 0.0033 & 0.3936 & 0.0188 & 0.1766 & -0.0184 & -0.0299 & -0.0623 \\
\hline a-SCop-MVN & 0.0022 & 0.2639 & 0.0191 & 0.1148 & -0.0183 & -0.0333 & -0.0613 \\
\hline a-SCop-MVT & 0.0020 & 0.2453 & 0.0201 & 0.0975 & -0.0209 & -0.0303 & -0.0673 \\
\hline DCop-MVN & 0.0024 & 0.2848 & 0.0191 & 0.1253 & -0.0180 & -0.0333 & -0.0645 \\
\hline DCop-MVT & 0.0024 & 0.2915 & 0.0203 & 0.1196 & -0.0207 & -0.0334 & -0.0718 \\
\hline ARMA-DCop-MVN & 0.0025 & 0.2968 & 0.0193 & 0.1298 & -0.0177 & -0.0334 & -0.0777 \\
\hline ARMA-DCop-MVT & 0.0027 & 0.3265 & 0.0192 & 0.1379 & -0.0163 & -0.0314 & -0.0707 \\
\hline VAR-DCop-MVN & 0.0026 & 0.3147 & 0.0189 & 0.1373 & -0.0194 & -0.0311 & -0.0670 \\
\hline a-DCop-MVN & 0.0024 & 0.2841 & 0.0191 & 0.1248 & -0.0118 & -0.0333 & -0.0644 \\
\hline a-DCop-MVT & 0.0024 & 0.2917 & 0.0203 & 0.1196 & -0.0207 & -0.0334 & -0.0717 \\
\hline
\end{tabular}

The factors are represented as

$$
f_{t}=H_{t}^{1 / 2} z_{t}
$$

where $H_{t}=E\left[f_{t} f_{t}^{\prime} \mid \mathscr{F}_{t-1}\right]$ is a diagonal matrix for all $t$ with elements $\left(h_{1 t}, \ldots, h_{n t}\right)$, which are the conditional variances of $f_{t}$. The symbol $z_{t}$ is $T \times 1$ vector of independent and identically distributed random errors such that $E\left[z_{t}\right]=0$ and $E\left[z_{t} z_{t}^{\prime}\right]=1$; implies that $E\left[f_{t} \mid \mathscr{F}_{t-1}\right]=0$ and $E\left[\epsilon_{t} \mid \mathscr{F}_{t-1}\right]=0$. The returns for the GO-GARCH model may be expressed as

$$
x_{t}=m+C H_{t}^{1 / 2} z_{t}
$$

For the conditional covariance matrix, it may be written as

$$
H_{t}=E\left[\left(x_{t}-m_{t}\right)\left(x_{t}-m_{t}\right)^{\prime} \mid \mathscr{F}_{t-1}\right]=C H_{t} C^{\prime}
$$

\subsubsection{Copula-GARCH (C-GARCH)}

We describe the multivariate random variable using a copula function to capture and model non-linear relationships between the asset returns. In the multivariate GARCH, the model assumes the stock and bond returns follow a multivariate normal or student distribution with linear correlation; and these assumptions are normally disregarded in many empirical finance studies. This study proposes various copula-GARCH based models considering the static version of copulas and dynamic copulas (more realistic in 


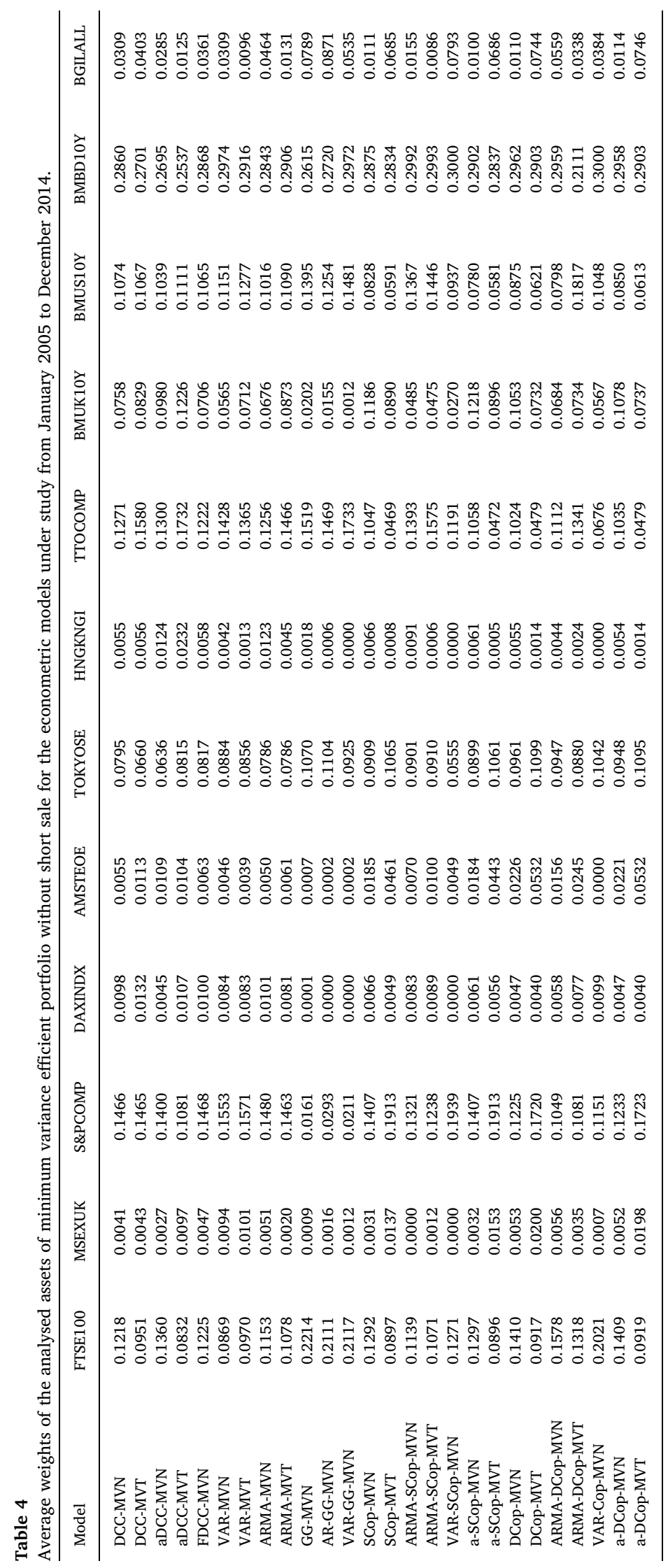


Table 5

Descriptive statistics and out-of-sample performance of minimum variance efficient portfolio with short sale for the econometric models under study from January 2005 to December 2014.

\begin{tabular}{|c|c|c|c|c|c|c|c|}
\hline Model & Return & Cumulative Return & Risk & Sharpe Ratio & VaR@90\% & VaR@95\% & VaR@99\% \\
\hline DCC-MVN & 0.0029 & 0.3444 & 0.0187 & 0.1611 & -0.0138 & -0.0219 & -0.0317 \\
\hline DCC-MVT & 0.0039 & 0.4670 & 0.0187 & 0.2148 & -0.0107 & -0.0160 & -0.0374 \\
\hline aDCC-MVN & 0.0038 & 0.4567 & 0.0187 & 0.2100 & -0.0114 & -0.0167 & -0.0462 \\
\hline aDCC-MVT & 0.0048 & 0.5734 & 0.0189 & 0.2603 & -0.0098 & -0.0163 & -0.0432 \\
\hline FDCC-MVN & 0.0029 & 0.3474 & 0.0189 & 0.1596 & -0.0139 & -0.0205 & -0.0317 \\
\hline VAR-MVN & 0.0029 & 0.3439 & 0.0185 & 0.1569 & -0.0139 & -0.0199 & -0.0409 \\
\hline VAR-MVT & 0.0027 & 0.3269 & 0.0181 & 0.1508 & -0.0118 & -0.0188 & -0.0404 \\
\hline ARMA-MVN & 0.0030 & 0.3588 & 0.0191 & 0.1644 & -0.0116 & -0.0186 & -0.0281 \\
\hline ARMA-MVT & 0.0034 & 0.4025 & 0.0184 & 0.1870 & -0.0114 & -0.0192 & -0.0392 \\
\hline GG-MVN & 0.0039 & 0.4676 & 0.0186 & 0.2127 & -0.0134 & -0.0228 & -0.0379 \\
\hline AR-GG-MVN & 0.0038 & 0.4513 & 0.0189 & 0.1980 & -0.0164 & -0.0226 & -0.0428 \\
\hline VAR-GG-MVN & 0.0035 & 0.4213 & 0.0183 & 0.1915 & -0.0125 & -0.0242 & -0.0364 \\
\hline SCop-MVN & 0.0020 & 0.2380 & 0.0184 & 0.1133 & -0.0199 & -0.0242 & -0.0534 \\
\hline SCop-MVT & 0.0018 & 0.2147 & 0.0205 & 0.0817 & -0.0194 & -0.0311 & -0.0624 \\
\hline ARMA-SCop-MVN & 0.0021 & 0.2525 & 0.0181 & 0.1174 & -0.0161 & -0.0289 & -0.0532 \\
\hline ARMA-SCop-MVT & 0.0021 & 0.2482 & 0.0178 & 0.1170 & -0.0169 & -0.0248 & -0.0550 \\
\hline VAR-SCop-MVN & 0.0035 & 0.4162 & 0.0172 & 0.2002 & -0.0167 & -0.0274 & -0.0610 \\
\hline a-SCop-MVN & 0.0019 & 0.2251 & 0.0184 & 0.1069 & -0.0193 & -0.0237 & -0.0559 \\
\hline a-SCop-MVT & 0.0018 & 0.2156 & 0.0205 & 0.0806 & -0.0203 & -0.0289 & -0.0645 \\
\hline DCop-MVN & 0.0017 & 0.2088 & 0.0187 & 0.0988 & -0.0166 & -0.0243 & -0.0630 \\
\hline DCop-MVT & 0.0016 & 0.1917 & 0.0211 & 0.0761 & -0.0205 & -0.0352 & -0.0793 \\
\hline ARMA-DCop-MVN & 0.0022 & 0.2689 & 0.0195 & 0.1188 & -0.0152 & -0.0206 & -0.0804 \\
\hline ARMA-DCop-MVT & 0.0024 & 0.2921 & 0.0188 & 0.1248 & -0.0164 & -0.0224 & -0.0642 \\
\hline VAR-Cop-MVN & 0.0027 & 0.3261 & 0.0186 & 0.1389 & -0.0201 & -0.0262 & -0.0487 \\
\hline a-DCop-MVN & 0.0017 & 0.2082 & 0.0187 & 0.0984 & -0.0166 & -0.0245 & -0.0624 \\
\hline a-DCop-MVT & 0.0016 & 0.1906 & 0.0211 & 0.0755 & -0.0205 & -0.0353 & -0.0794 \\
\hline
\end{tabular}

describing time-varying dependence structure between assets returns). We then examine the out-of-sample performance and compare with other models discussed earlier.

Let $F_{1}\left(x_{1}\right), \ldots, F_{n}\left(x_{n}\right)$ be the marginal distributions with a random vector $X=\left(x_{1}, \ldots, x_{n}\right)$. The random vector has uniform marginal distributions when we apply the probability integral transform to each of the component $\left(U_{1}, U_{2}, \ldots, U_{n}\right)=F_{1}\left(x_{1}\right), \ldots, F_{n}\left(x_{n}\right)$. Sklar (1959) showed that the copula can be depicted as

$$
F\left(x_{1}, \ldots, x_{n}\right)=C\left(F_{1}\left(x_{1}\right), \ldots, F_{n}\left(x_{n}\right)\right),
$$

such that $n$-dimensional copula $C\left(u_{1}, \ldots, u_{n}\right)$ is an $n$-dimensional random vector on $[0,1]^{d}$ with uniform marginals. The copula can be deduced from Eq. 26 as

$$
C\left(u_{1}, \ldots, u_{n}\right)=F\left(F_{1}^{-1}\left(u_{1}\right), F_{2}^{-1}\left(u_{2}\right), \ldots, F_{n}^{-1}\left(u_{n}\right)\right) .
$$

The copula $\left(X_{1}, X_{2}, \ldots, X_{n}\right)$ is defined as the joint cumulative distribution function of the continuous marginal distributions, which may be written as

$$
C\left(u_{1}, \ldots, u_{n}\right)=P\left[F_{1}\left(x_{1}\right) \leqslant u_{1}, F_{2}\left(X_{2}\right) \leqslant u_{2}, \ldots, F_{n}\left(x_{n}\right) \leqslant u_{n}\right] .
$$

The density function may be obtained as

$$
f\left(x_{1}, \ldots, x_{n}\right)=c\left(F_{1}\left(x_{1}\right), \ldots, F_{n}\left(x_{n}\right)\right) \prod_{i=1}^{n} f_{i}\left(x_{i}\right),
$$

such that $f_{i}$ are marginal densities and $F_{i}^{-1}$ is the quantile function of the margins. The density function of a copula is given by

$$
c\left(u_{i}, \ldots, u_{n}\right)=\frac{f\left(F_{i}^{-1}\left(u_{i}\right), \ldots, F_{n}^{-1}\left(u_{n}\right)\right)}{\prod_{i=1}^{n} f_{i}\left(F_{i}^{-1}\left(u_{i}\right)\right)}
$$

We use elliptical copulas which have been widely used in the literature for multivariate volatility modeling, i.e, Gaussian and Student- $t$ copulas. The $d$-dimensional Gaussian copula $C^{G A}\left(u_{1}, u_{2}, \ldots, u_{n}\right)$ is an $n$-dimensional distribution over the unit hypercube $[0,1]^{n}$ with uniform margins. The dependence structure is determined by the standardized correlation matrix $R$, such that the dispersion parameter $\rho_{1, n}$ is estimated using Kendall's $\tau$ method. The Gaussian copula is represented by

$$
C^{g}\left(u_{1}, u_{2}, \ldots, u_{n} ; R\right)=\int_{-\infty}^{\Phi^{-1}\left(u_{1}\right)} \ldots \int_{-\infty}^{\Phi^{-1}\left(u_{n}\right)} \frac{1}{2 \pi^{n / 2}|R|^{1 / 2}} \cdot e^{\left\{-\frac{1}{2} x^{\prime} R^{-1} x\right.} d_{1}, \ldots, d x_{n} .
$$




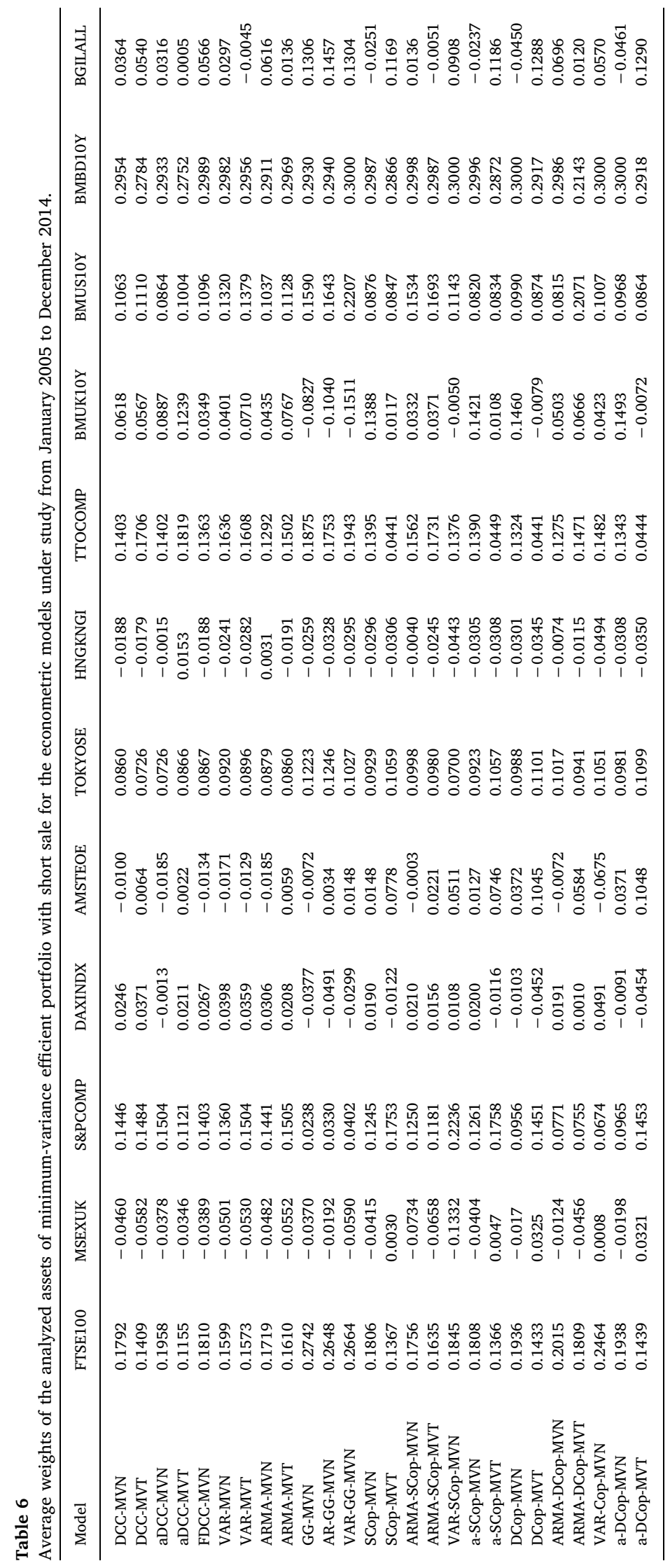


Table 7

Descriptive statistics and out-of-sample performance of mean-variance efficient portfolio without short sale for the econometric models under study from January 2005 to December 2014.

\begin{tabular}{|c|c|c|c|c|c|c|c|}
\hline Model & Return & Cumulative Return & Risk & Sharpe Ratio & VaR@90\% & VaR@95\% & VaR@99\% \\
\hline DCC-MVN & 0.0031 & 0.3672 & 0.0203 & 0.1530 & -0.0199 & -0.0240 & -0.0867 \\
\hline DCC-MVT & 0.0034 & 0.4099 & 0.0213 & 0.1528 & -0.0192 & -0.0338 & -0.0742 \\
\hline aDCC-MVN & 0.0033 & 0.4007 & 0.0192 & 0.1723 & -0.0137 & -0.0238 & -0.0627 \\
\hline aDCC-MVT & 0.0030 & 0.3629 & 0.0210 & 0.1356 & -0.0182 & -0.0288 & -0.0801 \\
\hline FDCC-MVN & 0.0031 & 0.1533 & 0.0203 & 0.1533 & -0.0199 & -0.0239 & -0.0867 \\
\hline VAR-MVN & 0.0038 & 0.4610 & 0.0200 & 0.1852 & -0.0195 & -0.0240 & -0.0723 \\
\hline VAR-MVT & 0.0036 & 0.4379 & 0.0205 & 0.1708 & -0.0183 & -0.0311 & -0.0745 \\
\hline ARMA-MVN & 0.0028 & 0.3400 & 0.0205 & 0.1457 & -0.0193 & -0.0258 & -0.0857 \\
\hline ARMA-MVT & 0.0022 & 0.2656 & 0.0189 & 0.1159 & -0.0174 & -0.0317 & -0.0681 \\
\hline GG-MVN & 0.0023 & 0.2816 & 0.0188 & 0.1282 & -0.0175 & -0.0295 & -0.0743 \\
\hline ARMA-GG-MVN & 0.0029 & 0.3440 & 0.0187 & 0.1534 & -0.0171 & -0.0293 & -0.0719 \\
\hline VAR-GG-MVN & 0.0021 & 0.2535 & 0.0184 & 0.1194 & -0.0202 & -0.0334 & -0.0697 \\
\hline SCop-MVN & 0.0022 & 0.2690 & 0.0191 & 0.1222 & -0.0189 & -0.0332 & -0.0610 \\
\hline SCop-MVT & 0.0022 & 0.2593 & 0.0201 & 0.1038 & -0.0202 & -0.0326 & -0.0666 \\
\hline ARMA-SCop-MVN & 0.0018 & 0.2157 & 0.0192 & 0.0991 & -0.0186 & -0.0332 & -0.0737 \\
\hline ARMA-SCop-MVT & 0.0021 & 0.2519 & 0.0186 & 0.1135 & -0.0189 & -0.0301 & -0.0699 \\
\hline VAR-SCop-MVN & 0.0033 & 0.3919 & 0.0201 & 0.1624 & -0.0211 & -0.0302 & -0.0589 \\
\hline a-SCop-MVN & 0.0022 & 0.2611 & 0.0191 & 0.1176 & -0.0194 & -0.0336 & -0.0598 \\
\hline a-SCop-MVT & 0.0022 & 0.2609 & 0.0201 & 0.1039 & -0.0205 & -0.0516 & -0.0645 \\
\hline DCop-MVN & 0.0026 & 0.3142 & 0.0194 & 0.1293 & -0.0160 & -0.0263 & -0.0674 \\
\hline DCop-MVT & 0.0024 & 0.2824 & 0.0202 & 0.1167 & -0.0208 & -0.0329 & -0.0717 \\
\hline ARMA-DCop-MVN & 0.0026 & 0.3075 & 0.0195 & 0.1210 & -0.0164 & -0.0270 & -0.0659 \\
\hline ARMA-DCop-MVT & 0.0027 & 0.3265 & 0.0192 & 0.1379 & -0.0163 & -0.0314 & -0.0707 \\
\hline VAR-DCop-MVN & 0.0023 & 0.2804 & 0.0189 & 0.1238 & -0.0180 & -0.0320 & -0.0671 \\
\hline a-DCop-MVN & 0.0026 & 0.3133 & 0.0194 & 0.1283 & -0.0164 & -0.0266 & -0.0672 \\
\hline a-DCop-MVT & 0.0024 & 0.2823 & 0.0202 & 0.1167 & -0.0207 & -0.0329 & -0.0717 \\
\hline
\end{tabular}

The density of the Gaussian copula, of the $d$-dimensional random vector $X$ may be written as in Bouye, Durrleman, and Roncalli (2000):

$$
\left.C^{g}\left(u_{1}, u_{2}, \ldots, u_{n} ; R\right)=\frac{1}{|R|^{1 / 2}} e^{\left\{-\frac{1}{2} \varsigma^{\prime}\left(R^{-1}-I\right) \varsigma\right.}\right\}
$$

where $\varsigma=\left(\phi^{-1}\left(u_{1}\right), \ldots, \phi^{-1}\left(u_{n}\right)\right)^{\prime}$ represents the quantile of the probability integral transformed (PIT) values of $X, f^{g}$ is the multivariate density of the normal distribution, $f_{i}$ is the density of the margin and $I$ is the identity matrix. The Gaussian copula is not able to account for tail dependence. Student- $t$ copula model allows for joint fat tails and an increased probability of joint extreme events as compared to Gaussian copula. This copula may be represented as

$$
C^{T}\left(u_{1}, u_{2}, \ldots, u_{n} ; R, v\right)=\int_{-\infty}^{t_{\nu}^{-1}\left(u_{1}\right)} \ldots \int_{-\infty}^{t_{\nu}^{-1}\left(u_{n}\right)} \frac{\Gamma\left(\frac{\nu+n}{2}\right)|R|^{-1 / 2}}{\Gamma\left(\frac{\nu}{2}\right)(\nu \pi)^{n / 2}} \cdot\left(1+\frac{1}{\nu} x^{\prime} R^{-1} x\right)^{-\frac{\nu+n}{2}} d x_{1} \ldots d x_{n}
$$

and the density of the Student- $t$ copula as

$$
C^{T}\left(u_{1}, u_{2}, \ldots, u_{n}\right)=|R|^{-1 / 2} \frac{\Gamma\left(\frac{v}{2}\right)}{\Gamma\left(\frac{v+1}{2}\right)}\left(\frac{\Gamma\left(\frac{v+n}{2}\right)}{\Gamma\left(\frac{v}{2}\right)}\right)^{n} \frac{\left(1+\frac{1}{\nu} \varsigma^{\prime} R^{-1} \varsigma\right)^{-\frac{\nu+n}{2}}}{\prod_{j=1}^{n}\left(1+\frac{\varsigma_{j}^{2}}{\nu}\right)^{-\frac{\nu-n}{2}}},
$$

where $\varsigma=\left(t_{v}^{-1}\left(u_{1}\right), \ldots, t_{v}^{-1}\left(u_{n}\right)\right)^{\prime}$. In Student- $t$ copula, the dependence structure introduces an additional parameter which is the degree of freedom $\nu$. As the value of $\nu$ increases, the tendency to exhibit extreme co-movements decreases. In our study, the copula-GARCH models are implemented using the multivariate Gaussian and Student- $t$ distributions, with static and dynamic (DCC) estimation of the correlation. The margins and PIT estimations are performed using a parametric density approach following Joe (1997). For the dependence measures, we use Kendall's $\tau$, as this method is based on order statistics of the sample which makes no assumption about the marginal distribution but depends only on copula $C$. Kendall's $\tau$ is defined as

$$
\tau\left(X_{i}, X_{j}\right)=\operatorname{Pr}\left[\left(X_{i}-X_{j}\right)-\left(Y_{i}-Y_{j}\right)>0\right]-\operatorname{Pr}\left[\left(X_{i}-X_{j}\right)-\left(Y_{i}-Y_{j}\right)<0\right],
$$

where $\left(X_{i}, Y_{i}\right)^{\prime}$ and $\left(X_{j}, Y_{j}\right)^{\prime}$ are vectors of random variables. Kendall's $\tau$ measures the difference between the probability of concordant and discordant pairs. The pairwise measure of concordance may be represented in terms of copula functions as

$$
\begin{aligned}
\tau\left(X_{i}, X_{j}\right) & =4 E\left[C\left(F_{i}\left(X_{i}\right), F_{j}\left(X_{j}\right)\right)\right]-1 \\
& =4 \int_{0}^{1} \int_{0}^{1} C\left(u_{i}, u_{j}\right) d C\left(u_{i}, u_{j}\right)-1 .
\end{aligned}
$$




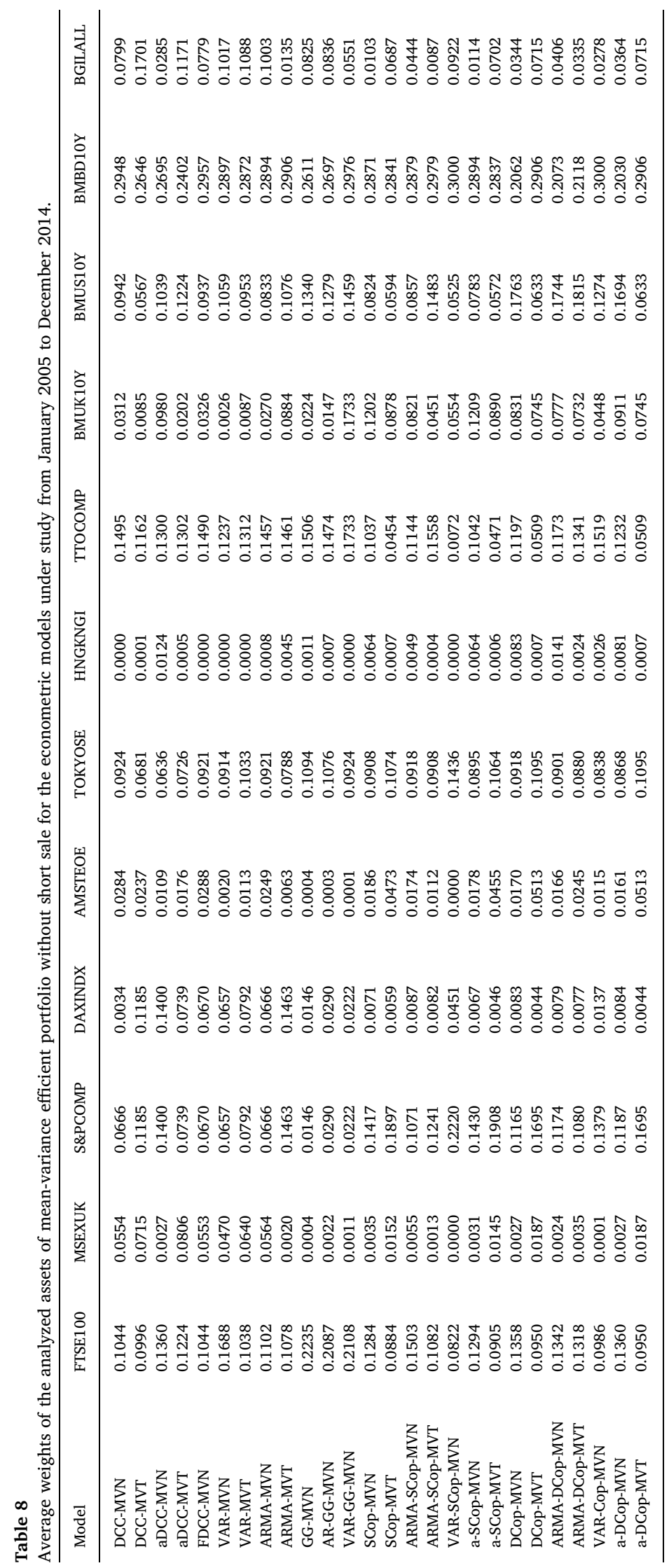


Table 9

Descriptive statistics and out-of-sample performance of mean-variance efficient portfolio with short sale for the econometric models under study from January 2005 to December 2014.

\begin{tabular}{|c|c|c|c|c|c|c|c|}
\hline Model & Return & Cumulative Return & Risk & Sharpe Ratio & VaR@90\% & VaR@95\% & VaR@99\% \\
\hline DCC-MVN & 0.0026 & 0.3081 & 0.0207 & 0.1286 & -0.0184 & -0.0287 & -0.1004 \\
\hline DCC-MVT & 0.0032 & 0.3837 & 0.0220 & 0.1336 & -0.0193 & -0.0370 & -0.0583 \\
\hline aDCC-MVN & 0.0038 & 0.4567 & 0.0187 & 0.2100 & -0.0114 & -0.0167 & -0.0462 \\
\hline aDCC-MVT & 0.0026 & 0.3095 & 0.0215 & 0.1095 & -0.0220 & -0.0270 & -0.0767 \\
\hline FDCC-MVN & 0.0026 & 0.3070 & 0.0207 & 0.1281 & -0.0184 & -0.0286 & -0.1004 \\
\hline VAR-MVN & 0.0038 & 0.4517 & 0.0208 & 0.1716 & -0.0148 & -0.0268 & -0.0789 \\
\hline VAR-MVT & 0.0038 & 0.4580 & 0.0209 & 0.1715 & -0.0169 & -0.0327 & -0.0646 \\
\hline ARMA-MVN & 0.0026 & 0.3123 & 0.0211 & 0.1568 & -0.0180 & -0.0279 & -0.0942 \\
\hline ARMA-MVT & 0.0021 & 0.2491 & 0.0184 & 0.1170 & -0.0160 & -0.0288 & -0.0555 \\
\hline GG-MVN & 0.0029 & 0.3497 & 0.0186 & 0.1558 & -0.0192 & -0.0303 & -0.0682 \\
\hline AR-GG-MVN & 0.0036 & 0.4297 & 0.0189 & 0.1916 & -0.0189 & -0.0278 & -0.0634 \\
\hline VAR-GG-MVN & 0.0030 & 0.3610 & 0.0183 & 0.1613 & -0.0191 & -0.0294 & -0.0646 \\
\hline SCop-MVN & 0.0020 & 0.2379 & 0.0184 & 0.1155 & -0.0197 & -0.0240 & -0.0550 \\
\hline SCop-MVT & 0.0018 & 0.2175 & 0.0206 & 0.0819 & -0.0198 & -0.0290 & -0.0640 \\
\hline ARMA-SCop-MVN & 0.0016 & 0.1861 & 0.0193 & 0.0871 & -0.0169 & -0.0263 & -0.0751 \\
\hline ARMA-SCop-MVT & 0.0023 & 0.2739 & 0.0178 & 0.1305 & -0.0176 & -0.0272 & -0.0544 \\
\hline VAR-SCop-MVN & 0.0042 & 0.5008 & 0.0192 & 0.2159 & -0.0206 & -0.0313 & -0.0634 \\
\hline a-SCop-MVN & 0.0019 & 0.2310 & 0.0184 & 0.1092 & -0.0200 & -0.0244 & -0.0522 \\
\hline a-SCop-MVT & 0.0019 & 0.2241 & 0.0205 & 0.0851 & -0.0197 & -0.0290 & -0.0629 \\
\hline DCop-MVN & 0.0023 & 0.2703 & 0.0189 & 0.1148 & -0.0146 & -0.0246 & -0.0604 \\
\hline DCop-MVT & 0.0015 & 0.1806 & 0.0211 & 0.0713 & -0.0210 & -0.0352 & -0.0791 \\
\hline ARMA-DCop-MVN & 0.0022 & 0.2652 & 0.0191 & 0.1112 & -0.0160 & -0.0225 & -0.0606 \\
\hline ARMA-DCop-MVT & 0.0024 & 0.2921 & 0.0188 & 0.1248 & -0.0164 & -0.0224 & -0.0642 \\
\hline VAR-Cop-MVN & 0.0024 & 0.2898 & 0.0181 & 0.1356 & -0.0171 & -0.0255 & -0.0677 \\
\hline a-DCop-MVN & 0.0023 & 0.2735 & 0.0189 & 0.1155 & -0.0145 & -0.0245 & -0.0610 \\
\hline a-DCop-MVT & 0.0015 & 0.1806 & 0.0211 & 0.0713 & -0.0210 & -0.0352 & -0.0791 \\
\hline
\end{tabular}

We also extend our analysis to include the dynamic copula models as investigated by Patton (2006), such that the vector stochastic process of financial returns $x_{t}=x_{i t}, \ldots, x_{n t}$ follows a copula-GARCH model with $\mu_{t}$ modeled as a time series model given by

$$
F\left(x_{t} \mid \mu_{t}, h_{t}\right)=C\left(F_{1}\left(x_{1 t} \mid \mu_{1 t}, h_{1 t}\right), \ldots, F_{n}\left(x_{n t} \mid \mu_{n t}, h_{n t}\right)\right) .
$$

Similar to other DCC models, the conditional variances $H_{t}$ can be estimated separately by a simple univariate GARCH specification. For the conditional density, it is given by

$$
C_{t}\left(u_{i t}, \ldots, u_{n t} \mid R, \eta\right)=\frac{f_{t}\left(F_{i}^{-1}\left(u_{i t} \mid \eta\right), \ldots, F_{n}^{-1}\left(u_{n t}\right) \mid R, \eta\right)}{\prod_{i=1}^{n} f_{i}\left(F_{i}^{-1}\left(u_{i t} \mid \eta\right) \mid \eta\right)}
$$

where $u_{i t}=F_{i t}\left(r_{i} t \mid \mu_{i t}, h_{i t}, \xi_{i}, v_{i}\right)$ is the PIT transformation of each series, which are estimated using parametric approach via the first stage GARCH process. The symbol $F_{i}^{-1}\left(u_{i t} \mid \eta\right)$ represents the quantile function of the margins subject to the common shape parameter $\nu$ of the multivariate density function.

The inputs in this study are efficient (since large number of models is employed for the estimation) and necessary as they are needed to perform the optimization procedure.

\section{Application to portfolio optimization}

One of the earliest approaches of the portfolio theory was developed by Markowitz (1952); a well-known approach known as a mean-variance optimization. It is a myopic strategy which assumes that the decision maker has a mean-variance criterion defined over the single period rate of return on the portfolio. Other related studies are Sharpe and Tint (1990), Engle and Colacito (2006), Platanakis and Sutcliffe (2014), Santos and Moura (2014). Rockafellar and Uryasev (2000), Bogentoft, Romeijn, and Uryasev (2001) and Alexander, Coleman, and Li (2006) have approached the optimization problem using the minimization of mean Conditional Value at Risk (CVaR). Recently, Boubaker and Sghaier (2013) and Huang and Hsu (2015) have proposed minimizing the CVaR assuming that the dependence structure is modeled by the copula parameter.

We employ five different optimization strategies: mean-variance, maximizing Sharpe ratio, mean-CVaR, and maximizing Sortino ratio. Here, we construct efficient portfolios with and without short sales, which sets box and group constraints on the weights such that the weights for each asset and the weights of groups of selected assets are restricted by lower and upper bounds, i.e., (i) portfolio without short sales, where no more than $30 \%$ is invested in each asset (i.e., $0 \leq x_{i} \leq 0.3, i=1, \ldots, n$ ), and (ii) portfolio with short sales, where a percentage between $-0.3 \%$ to $0.3 \%$ is invested in each asset (i.e., $-0.3 \leq x_{i} \leq 0.3, i=1, \ldots, n$ ), and $50 \%$ is the maximum percentage to invest in bonds and stocks. 


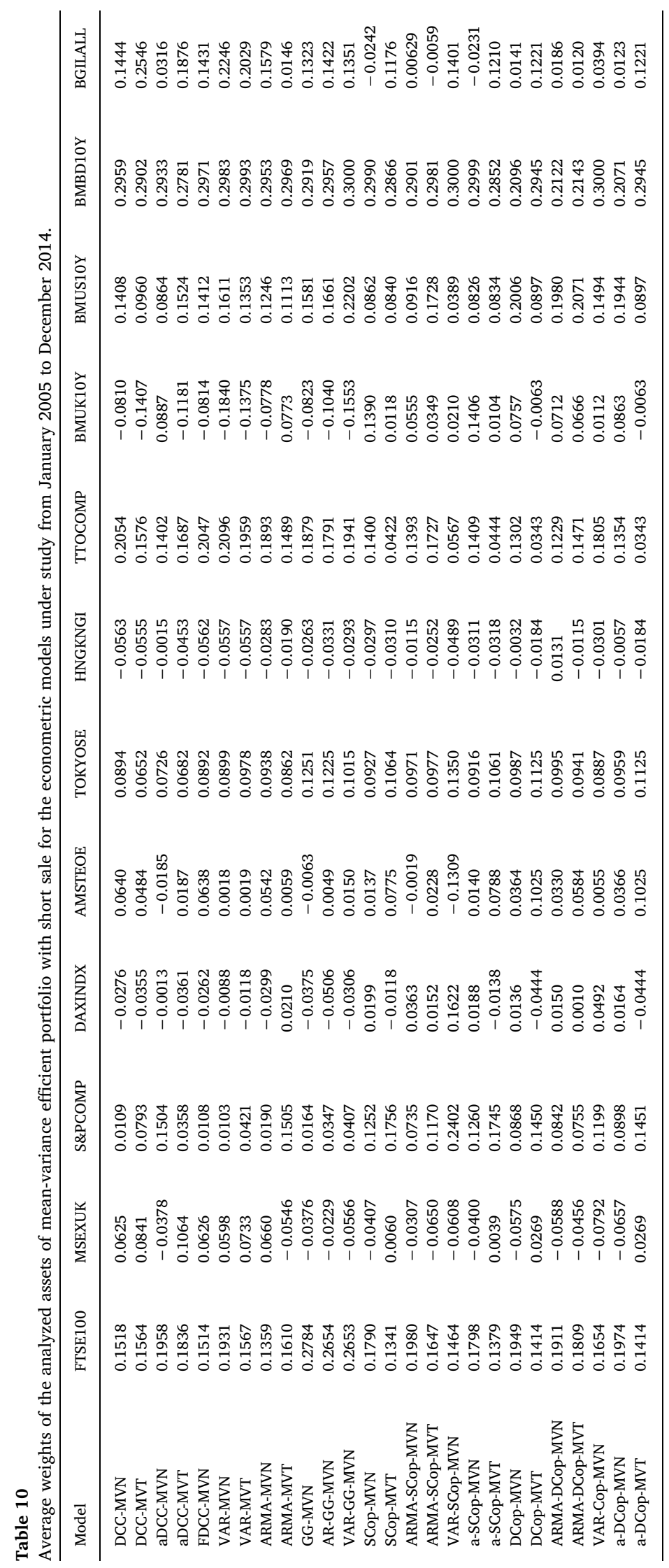


Table 11

Descriptive statistics and out-of-sample performance based on maximizing Sharpe ratio without short sale for the econometric models under study from January 2005 to December 2014.

\begin{tabular}{|c|c|c|c|c|c|c|c|}
\hline Model & Return & Cumulative Return & Risk & Sharpe Ratio & VaR@90\% & VaR@95\% & VaR@99\% \\
\hline DCC-MVN & 0.0041 & 0.4979 & 0.0235 & 0.1843 & -0.0181 & -0.0298 & -0.0625 \\
\hline DCC-MVT & 0.0049 & 0.5863 & 0.0239 & 0.1507 & -0.0169 & -0.0273 & -0.0698 \\
\hline aDCC-MVN & 0.0042 & 0.5091 & 0.0246 & 0.1796 & -0.0156 & -0.0320 & -0.0706 \\
\hline aDCC-MVT & 0.0053 & 0.6311 & 0.0248 & 0.2111 & -0.0205 & -0.0274 & -0.0796 \\
\hline FDCC-MVN & 0.0042 & 0.5047 & 0.0236 & 0.1864 & -0.0181 & -0.0300 & -0.0625 \\
\hline VAR-MVN & 0.0021 & 0.2469 & 0.0241 & 0.0783 & -0.0212 & -0.0400 & -0.0861 \\
\hline VAR-MVT & 0.0023 & 0.2744 & 0.0240 & 0.0870 & -0.0229 & -0.0400 & -0.0846 \\
\hline ARMA-MVN & 0.0075 & 0.9058 & 0.0243 & 0.3078 & -0.0194 & -0.0275 & -0.0735 \\
\hline ARMA-MVT & 0.0086 & 1.0312 & 0.0230 & 0.3474 & -0.0153 & -0.0215 & -0.0518 \\
\hline GG-MVN & 0.0047 & 0.5622 & 0.0256 & 0.1801 & -0.0258 & -0.0351 & -0.0807 \\
\hline AR-GG-MVN & 0.0132 & 1.5850 & 0.0249 & 0.5195 & -0.0155 & -0.0263 & -0.0667 \\
\hline VAR-GG-MVN & 0.0024 & 0.2938 & 0.0239 & 0.0934 & -0.0247 & -0.0400 & -0.0835 \\
\hline SCop-MVN & 0.0035 & 0.4151 & 0.0191 & 0.1842 & -0.0191 & -0.0437 & -0.0689 \\
\hline SCop-MVT & 0.0034 & 0.4034 & 0.0251 & 0.1354 & -0.0220 & -0.0428 & -0.0674 \\
\hline ARMA-SCop-MVN & 0.0049 & 0.5856 & 0.0236 & 0.2066 & -0.0202 & -0.0417 & -0.0728 \\
\hline ARMA-SCop-MVT & 0.0046 & 0.5551 & 0.0226 & 0.2088 & -0.0202 & -0.0373 & -0.0826 \\
\hline VAR-SCop-MVN & 0.0047 & 0.5588 & 0.0243 & 0.1890 & -0.0247 & -0.0354 & -0.0551 \\
\hline a-SCop-MVN & 0.0033 & 0.4020 & 0.0249 & 0.1326 & -0.0177 & -0.0360 & -0.0875 \\
\hline a-SCop-MVT & 0.0035 & 0.4236 & 0.0251 & 0.1409 & -0.0212 & -0.0413 & -0.0686 \\
\hline DCop-MVN & 0.0038 & 0.4571 & 0.0248 & 0.1508 & -0.0181 & -0.0426 & -0.0915 \\
\hline DCop-MVT & 0.0032 & 0.3844 & 0.0252 & 0.1318 & -0.0189 & -0.0406 & -0.0730 \\
\hline ARMA-DCop-MVN & 0.0035 & 0.4221 & 0.0236 & 0.1465 & -0.0193 & -0.0416 & -0.0794 \\
\hline ARMA-DCop-MVT & 0.0040 & 0.4763 & 0.0242 & 0.1608 & -0.0250 & -0.0436 & -0.0700 \\
\hline VAR-Cop-MVN & 0.0043 & 0.5184 & 0.0244 & 0.1906 & -0.0223 & -0.0341 & -0.0763 \\
\hline a-DCop-MVN & 0.0040 & 0.4768 & 0.0248 & 0.1567 & -0.0166 & -0.0385 & -0.0982 \\
\hline a-DCop-MVT & 0.0030 & 0.3566 & 0.0253 & 0.1245 & -0.0196 & -0.0403 & -0.0700 \\
\hline
\end{tabular}

\subsection{Minimum-variance}

The asset returns are assumed to be normally distributed and each investor wants to maximize their portfolio return at a minimal risk. This portfolio problem involves quadratic programming with linear constraints such that we want to construct an efficient portfolio with the lowest possible risk. We determine the optimal proportion allocation $w_{i}$ to the ith asset, where

$$
\sum_{i=1}^{n} w_{i}=1
$$

with the returns $x_{i}$ such that $i=1,2, \ldots, N$, and $x_{i} \sim N\left(\mu_{i}, \sigma_{i}^{2}\right)$ is an independent and identically distributed random vector. To characterize the portfolio, the expected return of a portfolio is written as

$$
E\left[R_{p}\right]=\sum_{i=1}^{n} x_{i} w_{i},
$$

and the variance of the portfolio is given by

$$
\sigma_{p}^{2}=\sum_{i=1}^{n} \sum_{j=1}^{n} \sigma_{i j} w_{i} w_{j}=w^{T} Q w
$$

where $Q$ is a $T \times n$ estimate of the covariance matrix of the assets returns. The vector $w$ denotes the weight of the asset, subject to the condition of $0 \leq w_{i} \leq 1$ for portfolio without short sales. To solve the optimization model, we choose to construct a portfolio of minimal risk

$$
\text { Minimize } w^{T} Q w
$$

subject to $R_{p}=\sum_{i=1}^{n} r_{i} w_{i}$ and $\sum_{i=1}^{n} w_{i}=1$.

\subsection{Mean-variance}

In a mean-variance optimization model, we want to construct an efficient portfolio with the lowest possible risk such that the return of the portfolio is greater than the target return. The mean-variance portfolios is constructed based on Eq. (40), subject to $R_{p}=\sum_{i=1}^{n} r_{i} w_{i}, E\left(R_{p}\right) \geqslant r_{\text {target }}$ and $\sum_{i=1}^{n} w_{i}=1$. 


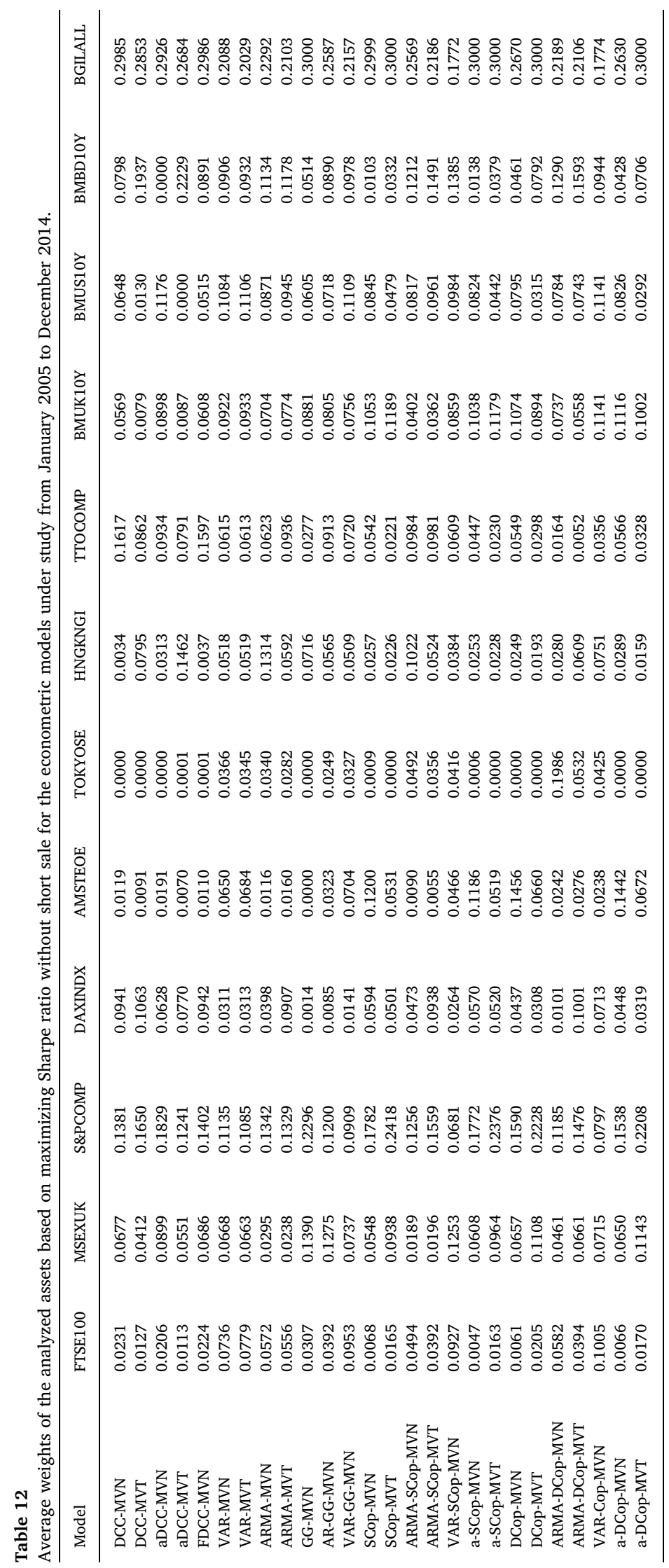


Table 13

Descriptive statistics and out-of-sample performance based on maximizing Sharpe ratio with short sale for the econometric models under study from January 2005 to December 2014.

\begin{tabular}{|c|c|c|c|c|c|c|c|}
\hline Model & Return & Cumulative Return & Risk & Sharpe Ratio & VaR@90\% & VaR@95\% & VaR@99\% \\
\hline DCC-MVN & 0.0047 & 0.5592 & 0.0249 & 0.1953 & -0.0240 & -0.0414 & -0.0734 \\
\hline DCC-MVT & 0.0064 & 0.7621 & 0.0252 & 0.2483 & -0.0137 & -0.0290 & -0.0420 \\
\hline aDCC-MVN & 0.0055 & 0.6627 & 0.0285 & 0.1932 & -0.0179 & -0.0321 & -0.0545 \\
\hline aDCC-MVT & 0.0072 & 0.8675 & 0.0270 & 0.2707 & -0.0161 & -0.0254 & -0.0519 \\
\hline FDCC-MVN & 0.0046 & 0.5510 & 0.0247 & 0.1945 & -0.0130 & -0.0221 & -0.0554 \\
\hline VAR-MVN & 0.0042 & 0.5033 & 0.0263 & 0.1608 & -0.0196 & -0.0306 & -0.0713 \\
\hline VAR-MVT & 0.0043 & 0.5216 & 0.0263 & 0.1681 & -0.0181 & -0.0312 & -0.0702 \\
\hline ARMA-MVN & 0.0159 & 1.9050 & 0.0263 & 0.5867 & -0.0073 & -0.0144 & -0.0362 \\
\hline ARMA-MVT & 0.0159 & 1.9046 & 0.0259 & 0.5961 & -0.0039 & -0.0145 & -0.0292 \\
\hline GG-MVN & 0.0061 & 0.7293 & 0.0279 & 0.2096 & -0.0247 & -0.0402 & -0.0566 \\
\hline AR-GG-MVN & 0.0244 & 2.9290 & 0.0263 & 0.9171 & -0.0032 & -0.0101 & -0.0289 \\
\hline VAR-GG-MVN & 0.0046 & 0.5485 & 0.0264 & 0.1748 & -0.0238 & -0.0364 & -0.0734 \\
\hline SCop-MVN & 0.0027 & 0.3229 & 0.0184 & 0.1508 & -0.0211 & -0.0524 & -0.0764 \\
\hline SCop-MVT & 0.0025 & 0.2992 & 0.0277 & 0.0965 & -0.0257 & -0.0513 & -0.0758 \\
\hline ARMA-SCop-MVN & 0.0053 & 0.6332 & 0.0258 & 0.2224 & -0.0219 & -0.0383 & -0.0698 \\
\hline ARMA-SCop-MVT & 0.0064 & 0.7665 & 0.0254 & 0.2668 & -0.0223 & -0.0423 & -0.0632 \\
\hline VAR-SCop-MVN & 0.0075 & 0.8961 & 0.0247 & 0.2939 & -0.0177 & -0.0246 & -0.0496 \\
\hline a-SCop-MVN & 0.0026 & 0.3094 & 0.0272 & 0.1013 & -0.0188 & -0.0497 & -0.0953 \\
\hline a-SCop-MVT & 0.0030 & 0.3620 & 0.0278 & 0.1098 & -0.0246 & -0.0471 & -0.0755 \\
\hline DCop-MVN & 0.0034 & 0.4033 & 0.0277 & 0.1143 & -0.0174 & -0.0452 & -0.1005 \\
\hline DCop-MVT & 0.0029 & 0.3462 & 0.0284 & 0.1089 & -0.0281 & -0.0496 & -0.0810 \\
\hline ARMA-DCop-MVN & 0.0045 & 0.5428 & 0.0262 & 0.1604 & -0.0210 & -0.0434 & -0.0705 \\
\hline ARMA-DCop-MVT & 0.0044 & 0.5320 & 0.0281 & 0.1516 & -0.0221 & -0.0489 & -0.0718 \\
\hline VAR-Cop-MVN & 0.0042 & 0.5087 & 0.0260 & 0.1802 & -0.0262 & -0.0344 & -0.0748 \\
\hline a-DCop-MVN & 0.0033 & 0.4000 & 0.0277 & 0.1180 & -0.0222 & -0.0403 & -0.1063 \\
\hline a-DCop-MVT & 0.0026 & 0.3175 & 0.0284 & 0.0997 & -0.0279 & -0.0500 & -0.0816 \\
\hline
\end{tabular}

\subsection{Maximizing the Sharpe ratio}

Now, we consider another alternative of optimization strategy; instead of minimizing the risk, we maximize the Sharpe ratio for a given risk free rate $r_{f}$. We consider

$$
\text { Maximize } \frac{\widehat{\mu}^{T} w-r_{f}}{w^{T} Q w},
$$

subject to $R_{p}=\sum_{i=1}^{n} x_{i} w_{i}$ and $\sum_{i=1}^{n} w_{i}=1$. The $p$-dimensional vector $\hat{\mu}$ is the estimates of the expected mean of the assets.

\subsection{Mean-CVaR}

Given a confidence level $\beta$ and a fixed $x \in X$, VaR is defined as the smallest number $l$ such that the probability of a loss $L$ is not more than $1-\beta$ for losses greater than $l$ :

$$
\operatorname{VaR}_{\beta}(x)=\inf \{l \in \mathbb{R}: P(L>l) \leqslant 1-\beta\}=\inf \left\{l \in \mathbb{R}: F_{L}(l) \geqslant \beta\right\},
$$

where $F_{L}$ is the distribution function of the losses. The mean-CVaR optimisation model is formulated as

$$
\text { Minimize } \operatorname{CVaR}_{\beta}(x)=\operatorname{Minimize} E\left[R \mid R \leqslant \operatorname{VaR}_{\beta}(x)\right] \text {, }
$$

such that $R \geq r_{\text {target }}$.

\subsection{Maximization of the Sortino ratio}

The last optimization strategy is the Sortino ratio, which is a modification of Sharpe ratio. This ratio uses downside deviation rather than standard deviation as the measure of risk. The Sortino ratio $S$ is defined as the ratio of the target return lowered by the risk-free rate and the CVaR risk. The risk-free rate is set at $r_{f}=0$.

\section{Data and empirical results}

We consider 12 assets consisting of eight stocks and four bond indices of 10 years maturity, in the United States (US), United Kingdom (UK), Germany, Japan, Netherlands, Canada and Hong Kong. In particular, our data set consists of monthly observations on eight stock indices: FTSE100, MSCI Europe Excluding UK (MSEXUK), S\&P 500 composite (S.PCOMP), DAX30 (DAXINDX), AEX (AMSTEOE), TOPIX (TOKYOSE), Hang Seng (HNGKNGI), and TSX composite (TTOCOMP). We also consider four bond indices: UK Benchmark 10-Year Government (BMUK10Y), US Benchmark 10-Year Government (BMUS10Y), Germany Benchmark 10-Year 


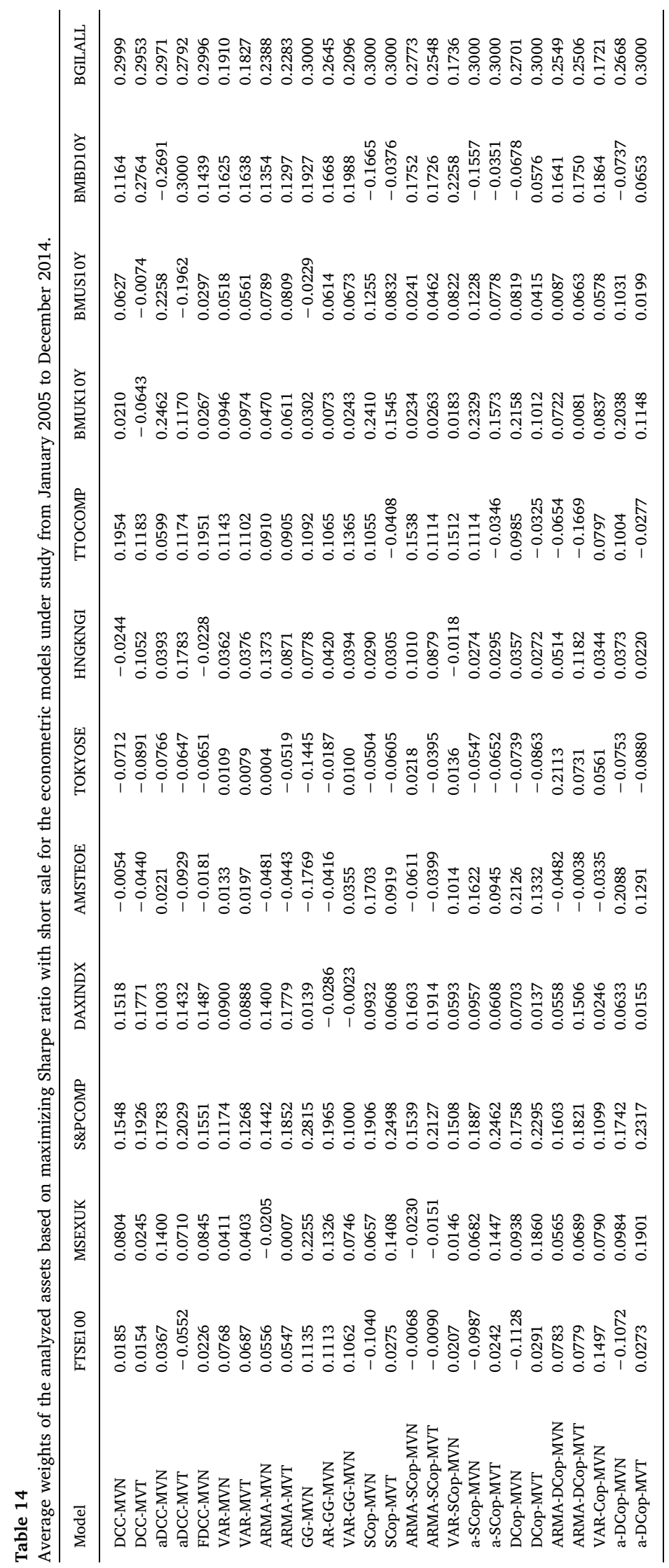


Table 15

Descriptive statistics and out-of-sample performance based on Mean-CVaR without short sale for the econometric models under study from January 2005 to December 2014.

\begin{tabular}{|c|c|c|c|c|c|c|c|}
\hline Model & Return & Cumulative Return & Risk & Sharpe Ratio & VaR@90\% & VaR@95\% & VaR@99\% \\
\hline DCC-MVN & 0.0158 & 1.8981 & 0.0226 & 0.6754 & -0.0090 & -0.0184 & -0.0495 \\
\hline DCC-MVT & 0.0020 & 0.2420 & 0.0197 & 0.1071 & -0.0214 & -0.0319 & -0.0645 \\
\hline aDCC-MVN & 0.0175 & 2.1035 & 0.0227 & 0.7545 & -0.0068 & -0.0156 & -0.0492 \\
\hline aDCC-MVT & 0.0022 & 0.2663 & 0.0181 & 0.1147 & -0.0181 & -0.0256 & -0.0622 \\
\hline FDCC-MVN & 0.0104 & 1.2471 & 0.0210 & 0.4620 & -0.0144 & -0.0224 & -0.0581 \\
\hline VAR-MVN & 0.0147 & 1.7618 & 0.0226 & 0.6176 & -0.0110 & -0.0288 & -0.0683 \\
\hline VAR-MVT & 0.0102 & 1.2266 & 0.0230 & 0.4409 & -0.0011 & -0.0208 & -0.0766 \\
\hline ARMA-MVN & 0.0173 & 2.0812 & 0.0227 & 0.7163 & -0.0086 & -0.0160 & -0.0439 \\
\hline ARMA-MVT & 0.0118 & 1.4152 & 0.0214 & 0.4847 & -0.0114 & -0.0208 & -0.0621 \\
\hline GOGARCH-MVN & 0.0183 & 2.1956 & 0.0232 & 0.7568 & -0.0047 & -0.0137 & -0.0504 \\
\hline AR-GOGARCH-MVN & 0.0150 & 1.7944 & 0.0230 & 0.6033 & -0.0124 & -0.0207 & -0.0513 \\
\hline VAR-GOGARCH-MVN & 0.0167 & 1.9996 & 0.0234 & 0.6979 & -0.0099 & -0.0245 & -0.0554 \\
\hline SCop-MVN & 0.0023 & 0.2766 & 0.0233 & 0.1025 & -0.0262 & -0.0396 & -0.0886 \\
\hline SCop-MVT & 0.0057 & 0.6888 & 0.0207 & 0.2661 & -0.0174 & -0.0306 & -0.0648 \\
\hline ARMA-SCop-MVN & 0.0159 & 1.9050 & 0.0229 & 0.6513 & -0.0113 & -0.0188 & -0.0520 \\
\hline ARMA-SCop-MVT & 0.0099 & 1.1857 & 0.0207 & 0.4368 & -0.0104 & -0.0221 & -0.0510 \\
\hline VAR-SCop-MVN & 0.0150 & 1.7993 & 0.0230 & 0.6125 & -0.0091 & -0.0191 & -0.0728 \\
\hline a-SCop-MVN & 0.0142 & 1.7008 & 0.0221 & 0.6080 & -0.0111 & -0.0173 & -0.0514 \\
\hline a-SCop-MVT & 0.0062 & 0.7433 & 0.0208 & 0.2889 & -0.0170 & -0.0312 & -0.0671 \\
\hline DCop-MVN & 0.0033 & 0.4001 & 0.0225 & 0.1482 & -0.0223 & -0.0310 & -0.0771 \\
\hline DCop-MVT & 0.0032 & 0.3847 & 0.0210 & 0.1481 & -0.0193 & -0.0291 & -0.0634 \\
\hline ARMA-DCop-MVN & 0.0021 & 0.2483 & 0.0222 & 0.0953 & -0.0196 & -0.0309 & -0.0867 \\
\hline ARMA-DCop-MVT & 0.0033 & 0.3910 & 0.0236 & 0.1452 & -0.0209 & -0.0408 & -0.0833 \\
\hline VAR-Cop-MVN & 0.0033 & 0.4009 & 0.0242 & 0.1444 & -0.0289 & -0.0407 & -0.0840 \\
\hline a-DCop-MVN & 0.0031 & 0.3684 & 0.0225 & 0.1388 & -0.0216 & -0.0303 & -0.0802 \\
\hline a-DCop-MVT & 0.0030 & 0.3550 & 0.0211 & 0.1403 & -0.0210 & -0.0262 & -0.0810 \\
\hline
\end{tabular}

Government(BMBD10Y), and FTSE Britain Government Linked Bond (BGILALL). All time series data were collected from Datastream for 30 years from January 1985 to December 2014, yielding to 360 observations.

The historical monthly returns of these stocks from the preceding 240 months (from January 1985 to December 2004) are used as the in-sample period to estimate the models. We employ recursive forecast (expanding-window) approach, where we use the data from January 1985 to December 2004 to make the first estimation in January 2005, data from January 1985 to January 2005 to make the second estimation in February 2005, and so on. The monthly returns for the considered time period are shown in Fig. 1, from which we clearly see the presence of volatility clustering. It is also obvious that the volatility of the series changes over time.

Table 1 presents the basic statistical characteristics of the time series. There are substantial differences in the characteristics for each of the analyzed asset return series; the stock indices have high average returns with a high volatility, while the bond returns have relatively lower average returns and volatilities.

The kurtosis for all of the assets ranges from 0.4 to 8.9, indicating fat tails in the asset return distributions. For the skewness, most of the assets are negatively skewed, indicating a distribution with an asymmetric tail extending toward more negative values. The results indicate that the asset returns exhibit skewed distributions, large variance and they are not normally distributed, which means that a time-varying conditional volatility exists. A normal distribution must be symmetric with excess kurtosis of zero.

We also examine if there is evidence for serial correlations in the asset returns by using the Ljung-Box Q(m) test statistics for the rates of return $R_{t}$, absolute rates of return $\left|R_{t}\right|$ and squared rates of return $R_{t}^{2}$ (see Table 1 ). There is evidence for a high level of autocorrelation in the absolute returns and the squared returns in which the null hypothesis of no autocorrelation is rejected at $5 \%$ level of significance for almost all of the assets series.

To test for normality, we conducted Shapiro test and obtained a $p$-value of $2.2 e^{-16}$, which again rejects the null hypothesis, indicating that the distribution is not normal. Statistical tests on these data indicate that the hypotheses for normality cannot be accepted for the majority of the assets. We use the covariance matrix of the portfolio to quantify the deviation from the expected return and to capture the investment risk, given the standard deviations and the covariance, the correlation can be determined from $\rho_{i j}=\frac{\sigma_{i} \sigma_{j}}{\sigma i j}$. Table 2 reports the pairwise correlation coefficients for the analyzed asset returns.

It is evident that the correlations within the stock and bond indices are relatively high. However, the pairwise correlation coefficients for the remaining pair asset returns exhibit low to medium correlations, indicating a potential for risk diversification in the constructed portfolio. The results confirm the presence of the stylized facts such as heavy tails, volatility clustering and heteroskedasticity in the asset returns distributions. The multivariate GARCH models are suitable to use to deal with these kinds of data.

\subsection{Empirical analysis}

The objective of this study is to investigate which econometric model gives the best estimates for the asset return and covariances in constructing optimal portfolio. By using different econometric models, we expect that this approach will be able to capture 


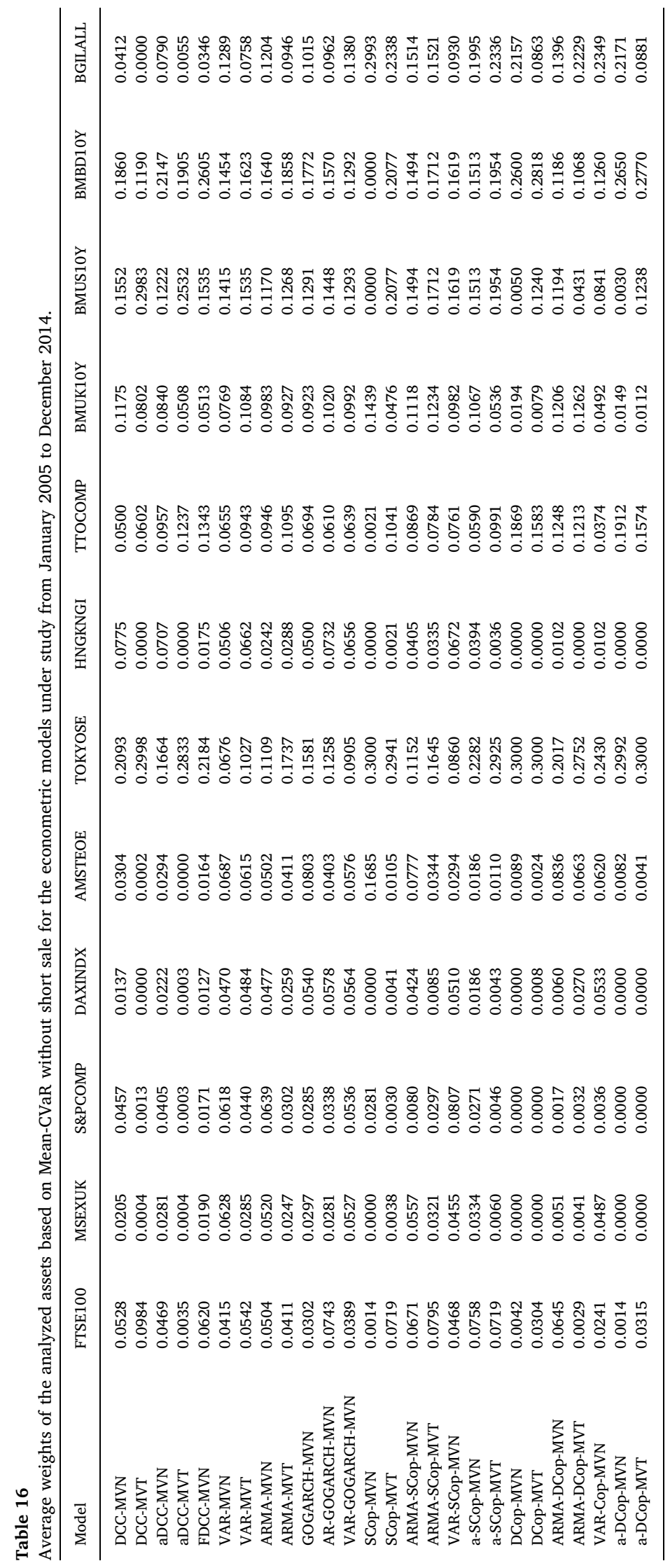


Table 17

Descriptive statistics and out-of-sample performance based on Mean-CVaR with short sale for the econometric models under study from January 2005 to December 2014.

\begin{tabular}{|c|c|c|c|c|c|c|c|}
\hline Model & Return & Cumulative Return & Risk & Sharpe Ratio & VaR@90\% & VaR@95\% & VaR@99\% \\
\hline DCC-MVN & 0.0465 & 5.5828 & 0.0294 & 1.5766 & 0.0200 & 0.0159 & 0.0027 \\
\hline DCC-MVT & 0.0399 & 4.7859 & 0.0266 & 1.4885 & 0.0135 & 0.0051 & 0.004 \\
\hline aDCC-MVN & 0.0463 & 5.5511 & 0.0293 & 1.5780 & 0.0187 & 0.0091 & 0.0028 \\
\hline aDCC-MVT & 0.0389 & 4.6720 & 0.0267 & 1.4563 & 0.0100 & 0.0045 & -0.0006 \\
\hline FDCC-MVN & 0.0446 & 5.3541 & 0.0287 & 1.5596 & 0.0187 & 0.0073 & 0.0000 \\
\hline VAR-MVN & 0.0467 & 5.5993 & 0.0314 & 1.4776 & 0.0197 & 0.0051 & -0.0056 \\
\hline VAR-MVT & 0.0341 & 4.0925 & 0.0296 & 1.1401 & 0.0000 & 0.0000 & 0.0000 \\
\hline ARMA-MVN & 0.0470 & 5.6432 & 0.0303 & 1.5548 & 0.0225 & 0.0123 & -0.0054 \\
\hline ARMA-MVT & 0.0443 & 5.3178 & 0.0293 & 1.5102 & 0.0194 & 0.0082 & 0.0014 \\
\hline GOGARCH-MVN & 0.0468 & 5.6163 & 0.0296 & 1.5741 & 0.0202 & 0.0096 & -0.0050 \\
\hline AR-GOGARCH-MVN & 0.0464 & 5.5669 & 0.0296 & 1.5603 & 0.0197 & 0.0081 & -0.0025 \\
\hline VAR-GOGARCH-MVN & 0.0473 & 5.6746 & 0.0315 & 1.4939 & 0.0228 & 0.0076 & -0.0068 \\
\hline SCop-MVN & 0.0037 & 0.4487 & 0.0293 & 0.1294 & -0.0350 & -0.0443 & -0.0739 \\
\hline SCop-MVT & 0.0408 & 4.8908 & 0.0271 & 1.5015 & 0.0177 & 0.0046 & -0.0019 \\
\hline ARMA-SCop-MVN & 0.0481 & 5.7749 & 0.0320 & 1.5049 & 0.0242 & 0.0088 & 0.0001 \\
\hline ARMA-SCop-MVT & 0.0428 & 5.1337 & 0.0285 & 1.4939 & 0.0171 & 0.0125 & -0.0027 \\
\hline VAR-SCop-MVN & 0.0477 & 5.7228 & 0.0305 & 1.5573 & 0.0249 & 0.0094 & 0.0019 \\
\hline a-SCop-MVN & 0.0462 & 5.5412 & 0.0292 & 1.5803 & 0.0233 & 0.0105 & -0.0019 \\
\hline a-SCop-MVT & 0.0410 & 4.9226 & 0.0273 & 1.4946 & 0.0171 & 0.0062 & -0.0068 \\
\hline DCop-MVN & 0.0041 & 0.4881 & 0.0292 & 0.1343 & -0.0334 & -0.0405 & -0.0771 \\
\hline DCop-MVT & 0.0045 & 0.5391 & 0.0307 & 0.1388 & -0.0350 & -0.0500 & -0.0806 \\
\hline ARMA-DCop-MVN & 0.0008 & 0.0926 & 0.0299 & 0.0144 & -0.0364 & -0.0450 & -0.1046 \\
\hline ARMA-DCop-MVT & 0.0033 & 0.3964 & 0.0317 & 0.1126 & -0.0363 & -0.0547 & -0.1073 \\
\hline VAR-Cop-MVN & 0.0041 & 0.4975 & 0.0292 & 0.1368 & -0.0353 & -0.0391 & -0.0625 \\
\hline a-DCop-MVN & 0.0365 & 4.3811 & 0.0263 & 1.4062 & 0.0121 & 0.0072 & 0.0000 \\
\hline a-DCop-MVT & 0.0045 & 0.5382 & 0.0306 & 0.1472 & -0.0325 & -0.0469 & -0.1015 \\
\hline
\end{tabular}

different characteristics in the data and will have economic impacts to optimal asset portfolio construction. As mentioned in Section 4, we split the data set of 360 observations period into 240 initial in-sample estimation period, for the parameter estimation and model selection to get the forecast for asset return and covariance matrix. Then, we have an initial out-of-sample period of 120 evaluation period, to evaluate the performance of the portfolio over the period. We generate each new forecast by adding new observations and re-estimating the model with the new observations as the data become available. We repeat the steps for each of the optimization strategies.

\subsection{Evaluating the out-of-sample portfolio performance}

To measure the performance of the portfolio, we evaluate 120 out-of-sample periods and perform the optimal asset allocation with a difference covariance estimator depending on the time period for each of the optimization models.

We repeat the exercise several times using 26 different model specifications while considering efficient portfolios with and without short sales: DCC GARCH Normal (DCC-MVN), DCC GARCH Student (DCC-MVT), Asymmetric DCC GJR-GARCH Normal (aDCC-MVN), Asymmetric DCC GJR-GARCH Student (aDCC-MVT), FDCC GARCH Normal (FDCC), VAR DCC GARCH Normal (VARMVN), VAR DCC GARCH Student (VAR-MVT), ARMA DCC GARCH Normal (ARMA-MVN), ARMA Student (ARMA-MVT), GOGARCHMVN (GG-MVN), ARMA GOGARCH-MVN (ARMA-GG-MVN), VAR GOGARCH-MVN (VAR-GG-MVN), Static Copula-MVN (SCopMVN), Static Copula-MVT (SCop-MVT), Static ARMA Copula-MVN (ARMA-SCop-MVN), Static ARMA Copula-MVT (ARMA-SCopMVT), Static VAR Copula-MVN (VAR-SCop-MVN), Static Asymmetric Copula-MVN (a-SCop-MVN), Static Asymmetric Copula-MVT (a-SCop-MVT), Dynamic Copula-MVN (DCop-MVN), Dynamic Copula-MVT (DCop-MVT), Dynamic ARMA Copula-MVN (ARMADCop-MVN), Dynamic ARMA Copula-MVT (ARMA-DCop-MVT), Dynamic VAR Copula-MVN (VAR-DCop-MVN), Dynamic Asymmetric Copula-MVN (a-DCop-MVN), Dynamic Asymmetric Copula-MVT (a-DCop-MVT).

\subsubsection{Efficient minimum variance portfolio without short sales}

Table 3 presents the out-of-sample portfolio performance of minimum variance portfolio without short sales and restricting the portfolio weights to be $0 \leq x_{i} \leq 0.3, i=1, \ldots, n$, and the investment of $50 \%$ in stocks and bonds. In general, the empirical results suggest that the dynamic models are able to deliver performance gains over the static models. The best model is the aDCC-MVT, which accrues significant average monthly return of $0.38 \%$, cumulative return of $45.48 \%$ and Sharpe ratio of 0.2013 . The next best model is the VAR-SCop-MVN, which recorded an average monthly return of $0.33 \%$, cumulative return of $39.36 \%$ and Sharpe ratio of 0.1766 , but with a lower risk $(1.88 \%)$ as compared to aDCC-MVT $(1.91 \%)$.

Table 4 presents the average weight of an efficient portfolio without short sales using the minimum variance. We observe that all models invested mainly in BMBD10Y which is a bond index, ranging from $20 \%$ to $30 \%$ out of the overall investment. The next biggest allocation is invested in stock indices, i.e., FTSE100, S\&PCOMP and TTOCOMP with around $10 \%$ to $22 \%$ of total investment. Note 


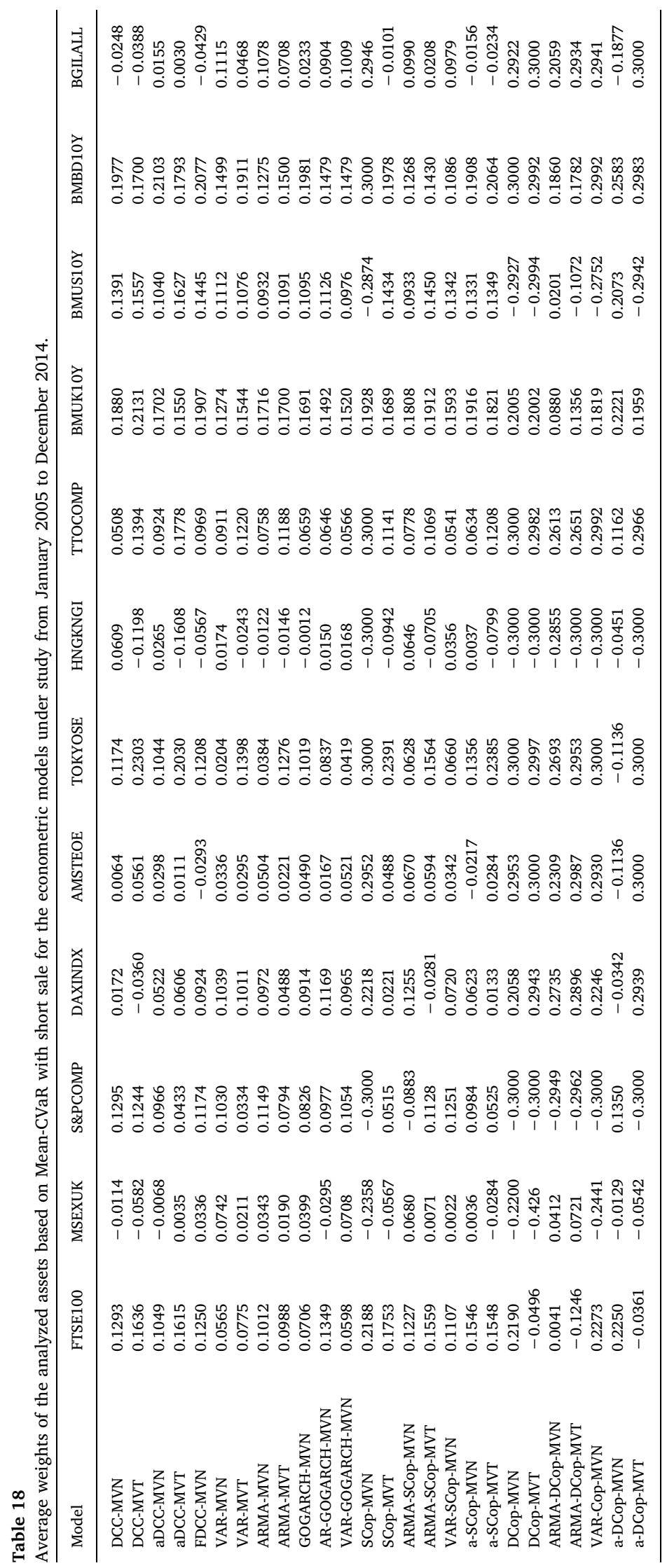


Table 19

Descriptive statistics and out-of-sample performance based on Maximizing Sortino ratio without short sale for the econometric models under study from January 2005 to December 2014.

\begin{tabular}{|c|c|c|c|c|c|c|c|}
\hline Model & Return & Cumulative Return & Risk & Sharpe Ratio & VaR@90\% & VaR@95\% & VaR@99\% \\
\hline DCC-MVN & 0.0171 & 2.0530 & 0.0233 & 0.7480 & 0.0000 & -0.0131 & -0.0440 \\
\hline DCC-MVT & 0.0194 & 2.3319 & 0.0231 & 0.8408 & 0.0000 & -0.0119 & -0.0440 \\
\hline aDCC-MVN & 0.0170 & 2.0407 & 0.0230 & 0.7624 & 0.0000 & -0.0129 & -0.0450 \\
\hline aDCC-MVT & 0.0191 & 2.2893 & 0.0228 & 0.8322 & 0.0000 & -0.0119 & -0.0397 \\
\hline FDCC-MVN & 0.0172 & 2.0604 & 0.0232 & 0.7497 & 0.0000 & -0.0131 & -0.0440 \\
\hline VAR-MVN & 0.0087 & 1.0493 & 0.0229 & 0.3914 & -0.0161 & -0.0420 & -0.0755 \\
\hline VAR-MVT & 0.0031 & 0.3677 & 0.0242 & 0.1460 & -0.0178 & -0.0466 & -0.0902 \\
\hline ARMA-MVN & 0.0110 & 1.3225 & 0.0228 & 0.4989 & 0.0000 & -0.0156 & -0.0647 \\
\hline ARMA-MVT & 0.0137 & 1.6495 & 0.0225 & 0.6074 & 0.0000 & -0.0139 & -0.0438 \\
\hline GOGARCH-MVN & 0.0155 & 1.8607 & 0.0232 & 0.6801 & 0.0000 & -0.0128 & -0.0448 \\
\hline AR-GOGARCH-MVN & 0.0149 & 1.7886 & 0.0229 & 0.6515 & 0.0000 & -0.0144 & -0.0576 \\
\hline VAR-GOGARCH-MVN & 0.0080 & 0.9571 & 0.0236 & 0.3316 & 0.0000 & -0.0254 & -0.0629 \\
\hline SCop-MVN & 0.0174 & 2.0886 & 0.0227 & 0.7775 & 0.0000 & -0.0128 & -0.0440 \\
\hline SCop-MVT & 0.0186 & 2.2354 & 0.0228 & 0.8038 & 0.0000 & -0.0119 & -0.0440 \\
\hline ARMA-SCop-MVN & 0.0118 & 1.4117 & 0.0226 & 0.5306 & -0.0011 & -0.0167 & -0.0775 \\
\hline ARMA-SCop-MVT & 0.0148 & 1.7713 & 0.0228 & 0.6398 & 0.0000 & -0.0128 & -0.0479 \\
\hline VAR-SCop-MVN & 0.0083 & 0.9946 & 0.0238 & 0.3338 & 0.0000 & -0.0200 & -0.0538 \\
\hline a-SCop-MVN & 0.0168 & 2.0194 & 0.0226 & 0.7585 & 0.0000 & -0.0115 & -0.0440 \\
\hline a-SCop-MVT & 0.0190 & 2.2784 & 0.0231 & 0.8197 & 0.0000 & -0.0119 & -0.0440 \\
\hline DCop-MVN & 0.0168 & 2.0131 & 0.0229 & 0.7536 & 0.0000 & -0.0128 & -0.0440 \\
\hline DCop-MVT & 0.0187 & 2.2452 & 0.0229 & 0.8165 & 0.0000 & -0.0119 & -0.0440 \\
\hline ARMA-DCop-MVN & 0.0104 & 1.2483 & 0.0221 & 0.4692 & 0.0000 & -0.0142 & -0.0618 \\
\hline ARMA-DCop-MVT & 0.0103 & 1.2419 & 0.0212 & 0.4978 & 0.0000 & -0.0156 & -0.0446 \\
\hline VAR-Cop-MVN & 0.0087 & 1.0434 & 0.0234 & 0.3681 & -0.0012 & -0.0173 & -0.0643 \\
\hline a-DCop-MVN & 0.0170 & 2.0385 & 0.0230 & 0.7669 & 0.0000 & -0.0128 & -0.0440 \\
\hline a-DCop-MVT & 0.0188 & 2.2518 & 0.0229 & 0.8177 & 0.0000 & -0.0119 & -0.0440 \\
\hline
\end{tabular}

that, most of the models allocate only a very small percentage to DAXINDX with only 0 to $1 \%$ out of total investments.

\subsubsection{Efficient minimum variance portfolio with short sales}

The results presented in Table 5 are based on the out-of-sample performance of the constructed minimum-variance efficient portfolio with short sales based on the constraints of the portfolio weights to be $-0.3 \leq x_{i} \leq 0.3, i=1, \ldots, n$ and the investment of $50 \%$ in stocks and bonds.

By using these constraints, we observe that the multivariate aDCC-MVT once again outperforms the other models. The aDCC-MVT has the highest cumulative return of $57.34 \%$ with the Sharpe ratio of 0.2603 , but at a higher risk of $1.89 \%$. On the other hand, the GOGARCH-MVN and DCC-MVT models produce a high average return at a lower risk. By incorporating the copula function, we can see that it does not bring any improvement to the model performance. For mean-variance portfolios with short sales, the GOGARCH, DCC and aDCC models perform well compared to the other models.

Similar to the portfolio without short sales, all models have invested mainly in bonds, i.e., the highest allocation is to asset BMBD10Y with about $30 \%$ of the investment, while a substantial fraction of the investment is allocated to stock indices (see Table 6). This is expected since bond indices are known to be 'safer' as they have medium volatilities with lower average returns. If the investor is risk-averse, then he will take the risk of investing in a higher risk portfolio. Note that the allocation to DAXINDX, AMSTEOE, HNGKNGI, BMUK10Y, and BGILALL are different across the models.

\subsubsection{Efficient mean-variance portfolio without short sales}

By using the same constraints, we obtained the results of the out-of-sample performance of mean-variance optimization without short sales, as presented in Table 7 . Given a target return $r_{\text {target }}=0.33 \%$, we observe that the multivariate DCC-MVT model has the highest cumulative return of $40.99 \%$ and achieved a higher Sharpe ratio of 0.1528 . Next, we present the average weight of an efficient portfolio without short sales using the mean-variance optimization. On the basis of the results in Table 8, similar to minimum-variance portfolios, we observe that all models invested mainly in the bond index BMBD10Y, ranging from $26 \%$ to $30 \%$ out of the overall investment. FTSE100 also has a high allocation at around $10 \%$ to $22 \%$ across all models. Most of the models do not invest in stock HNGKNGI, which has high volatility, while some models allocate only a small proportion, i.e., around $1 \%$.

\subsubsection{Efficient mean-variance portfolio with short sales}

The results for the optimal mean-variance portfolios with the weights constraints of $-0.3 \leq x_{i} \leq 0.3, i=1, \ldots, n$, and a target expected return of $0.34 \%$, are presented in Table 9 . We can see that the multivariate aDCC-MVT, VAR-MVT and VAR-MVN models outperform the other competing models. In general, these models have higher Sharpe ratios, i.e., 0.2100 (aDCC-MVN), 0.1716 (VARMVN) and 0.1715 (VAR-MVT). These models also have lower values of the corresponding risk measures such as the volatility and the value at risk values. Table 10 . 


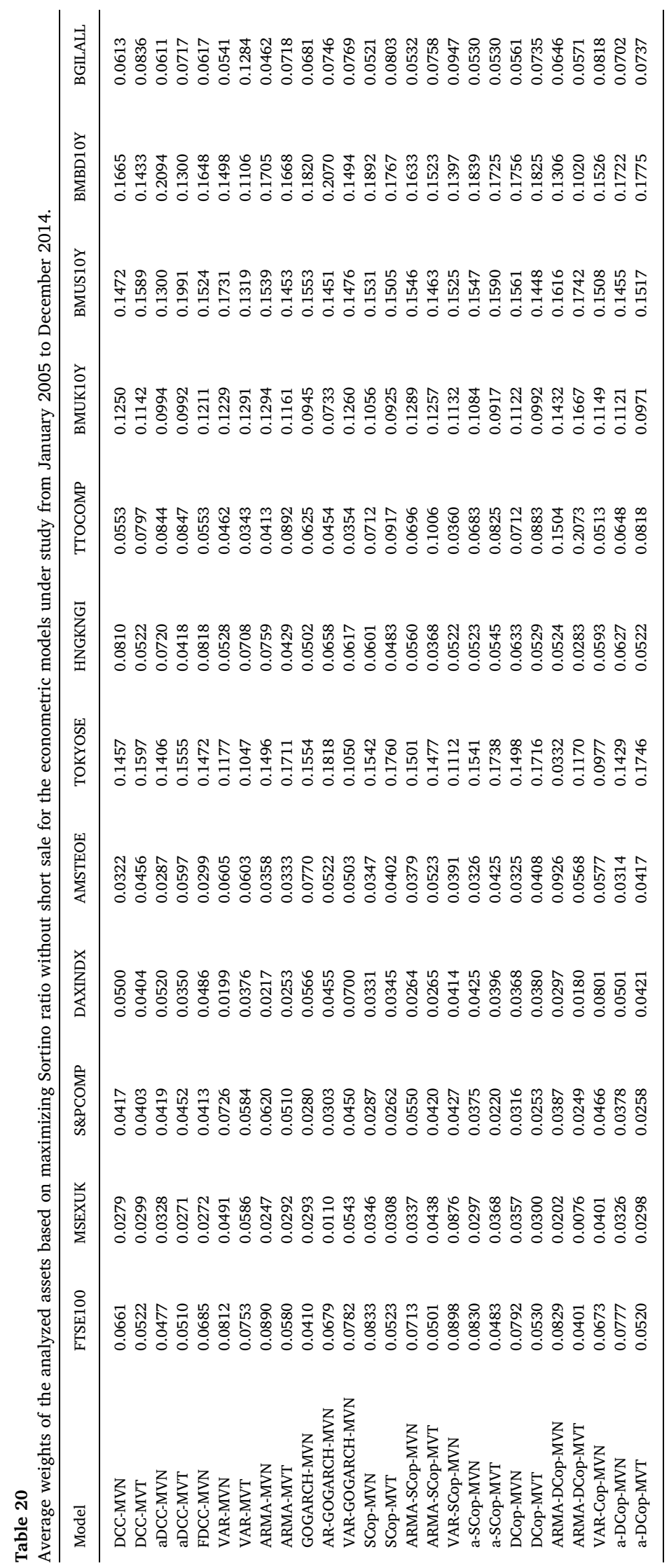


Table 21

Descriptive statistics and out-of-sample performance based on maximizing Sortino ratio with short sale for the econometric models under study from January 2005 to December 2014.

\begin{tabular}{|c|c|c|c|c|c|c|c|}
\hline Model & Return & Cumulative Return & Risk & Sharpe Ratio & VaR@90\% & VaR@95\% & VaR@99\% \\
\hline DCC-MVN & 0.0362 & 4.3470 & 0.0272 & 1.3446 & 0.0014 & 0.0007 & 0.0000 \\
\hline DCC-MVT & 0.0540 & 6.4811 & 0.0325 & 1.6599 & 0.0285 & 0.0172 & 0.0052 \\
\hline aDCC-MVN & 0.0333 & 3.9977 & 0.0263 & 1.2776 & 0.0090 & 0.0000 & 0.0000 \\
\hline aDCC-MVT & 0.0283 & 3.3954 & 0.0252 & 1.1447 & 0.0091 & 0.0000 & 0.0000 \\
\hline FDCC-MVN & 0.0369 & 4.4271 & 0.0274 & 1.3605 & 0.0140 & 0.0074 & 0.0000 \\
\hline VAR-MVN & 0.0268 & 3.2168 & 0.0292 & 0.9214 & 0.0000 & 0.0000 & 0.0000 \\
\hline VAR-MVT & 0.0164 & 1.9721 & 0.0304 & 0.5471 & 0.0000 & 0.0000 & 0.0000 \\
\hline ARMA-MVN & 0.0229 & 2.7496 & 0.0276 & 0.8444 & 0.0000 & 0.0000 & 0.0000 \\
\hline ARMA-MVT & 0.0149 & 1.7829 & 0.0259 & 0.5799 & 0.0000 & 0.0000 & 0.0000 \\
\hline GOGARCH-MVN & 0.0326 & 3.9096 & 0.0256 & 1.2733 & 0.0095 & 0.0000 & 0.0000 \\
\hline AR-GOGARCH-MVN & 0.0125 & 1.4958 & 0.0254 & 0.4953 & 0.0000 & 0.0000 & 0.0000 \\
\hline VAR-GOGARCH-MVN & 0.0294 & 3.5317 & 0.0303 & 0.9507 & 0.0000 & 0.0000 & 0.0000 \\
\hline SCop-MVN & 0.0359 & 4.3089 & 0.0262 & 1.3844 & 0.0114 & 0.0000 & 0.0000 \\
\hline SCop-MVT & 0.0339 & 4.0714 & 0.0257 & 1.3242 & 0.0122 & 0.0000 & 0.0000 \\
\hline ARMA-SCop-MVN & 0.0275 & 3.2972 & 0.0283 & 0.9672 & 0.0000 & 0.0000 & 0.0000 \\
\hline ARMA-SCop-MVT & 0.0231 & 2.7711 & 0.0265 & 0.8740 & 0.0000 & 0.0000 & 0.0000 \\
\hline VAR-SCop-MVN & 0.0317 & 3.8009 & 0.0301 & 1.0673 & 0.0000 & 0.0000 & 0.0000 \\
\hline a-SCop-MVN & 0.0362 & 4.3458 & 0.0263 & 1.3909 & 0.0123 & 0.0000 & 0.0000 \\
\hline a-SCop-MVT & 0.0339 & 4.0639 & 0.0259 & 1.3170 & 0.0142 & 0.0000 & 0.0000 \\
\hline DCop-MVN & 0.0358 & 4.2960 & 0.0261 & 1.3823 & -0.0117 & 0.0000 & 0.0000 \\
\hline DCop-MVT & 0.0333 & 3.9993 & 0.0257 & 1.3110 & -0.0130 & 0.0000 & 0.0000 \\
\hline ARMA-DCop-MVN & 0.0254 & 3.0434 & 0.0287 & 0.8573 & 0.0000 & 0.0000 & 0.0000 \\
\hline ARMA-DCop-MVT & 0.0197 & 2.3607 & 0.0253 & 0.7462 & 0.0000 & 0.0000 & 0.0000 \\
\hline VAR-Cop-MVN & 0.0283 & 3.3995 & 0.0300 & 0.9370 & 0.0000 & 0.0000 & 0.0000 \\
\hline a-DCop-MVN & 0.0365 & 4.3811 & 0.0263 & 1.4062 & 0.0072 & 0.0000 & 0.0000 \\
\hline a-DCop-MVT & 0.0329 & 3.9475 & 0.0259 & 1.2847 & 0.0000 & 0.0000 & 0.0000 \\
\hline
\end{tabular}

In general, when we allow short sales, we observe that all models short sell the stock HNGKNGI, ranging from $-1 \%$ to $-6 \%$. On the other hand, all models take long positions of their portfolios to FTSE100, S\&PCOMP, TOKYOSE, TTOCOMP, BMUK10Y, and BMUS10Y.

\subsubsection{Efficient portfolio based on maximizing Sharpe ratio without short sales}

In this section, we construct an optimal portfolio based on maximizing the Sharpe ratio for a portfolio without short sales. The results are presented in Table 11 assuming the risk-free rate $r_{f}$ to be $0 \%$. From the results, we observe that the multivariate AR-GGMVN model outperforms the other models, by having the highest value in three out of five portfolio measurements, with a high risk of 2.49\%. The other best optimal models are ARMA-MVT and ARMA-MVN. The results for the maximization of Sharpe ratio show that it has a better portfolio in terms of higher return and Sharpe ratio as compared to the mean-variance optimization. However, this optimization strategy has a higher risk in all of the models when compared to the mean-variance strategy.

Table 12 presents the average weight of an efficient portfolio without short sales based on maximizing the Sharpe ratio. In the mean-variance optimization, the biggest proportion of investment goes to BMBD10Y, while in maximizing the Sharpe ratio, all models invested mainly in a bond index BGILALL for about $20 \%$ to $30 \%$ of the overall investment.

\subsubsection{Efficient portfolio based on maximizing Sharpe ratio with short sales}

When we allow short selling, such that the constraints of the portfolio weights to be between $-0.3 \leq x_{i} \leq 0.3, i=1, \ldots, n$, we obtained a more attractive portfolio as we have higher monthly return, cumulative return and Sharpe ratio in most of the models as compared to the portfolio without short sales. The risk-free rate $r_{f}$ is assumed to be $0 \%$.

Table 13 presents the results obtained from constructing an efficient Sharpe ratio portfolio with short sales. From the results, we observe that, once again, the multivariate AR-GG-MVN, ARMA-MVN and ARMA-MVT models outperform the other models. The ARGG-MVN and ARMA-MVN models have the highest risk of $2.63 \%$ among all models, whereas ARMA-MVT has a slightly lower risk of $2.59 \%$.

The average weights of the efficient portfolios with short sales based on maximizing the Sharpe ratio are presented in Table 14. Similarly as with the portfolio without short sales, the biggest asset allocation goes to BGILALL for about $18 \%$ to $30 \%$ out of the total investment. Note that some assets have different allocations across the models.

\subsubsection{Efficient portfolio Mean-CVaR portfolio without short sales}

In the Mean-CVaR optimization, we compute the optimized efficient portfolio which has the lowest risk for a given return. The portfolio is constructed by minimizing the conditional value at risk using a 95\% probability level, with similar restrictions as in other models. When the portfolio is in the long position, the results are presented in Table 15. The aDCC-MVN model once again outperforms other models with the highest average return of $1.75 \%$, followed by ARMA-MVN, which recorded average return of $1.73 \%$ 


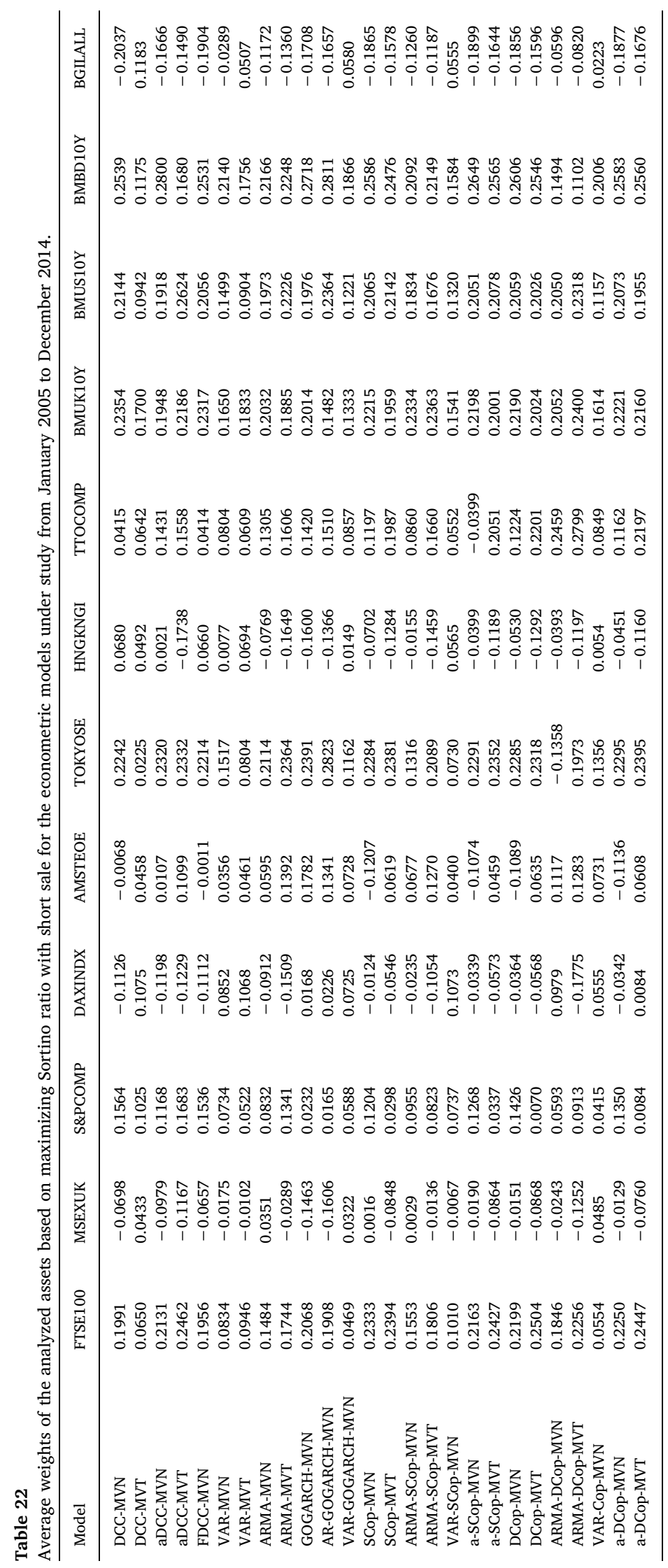


with a similar volatility of $2.27 \%$.

The highest asset allocation of this model mainly goes to stock TOKYOSE, which dominating the portfolio, for about $10 \%$ to $30 \%$ of total investment. The other allocations are invested in bond indices, i.e., BMBD10Y and BMUS10Y with around 8\% to 30\%. The average weights for the analyzed assets are presented in Table 16.

\subsubsection{Efficient portfolio mean-CVaR portfolio with short sales}

Table 17 reports the monthly average returns of the constructed portfolios, the cumulative returns, risk and value at risk at $90 \%$, 95\%, and 99\% probability levels. The portfolio allows short selling, that is the portfolio is constructed when we restrict the portfolio weights to be $-0.3 \leq x_{i} \leq 0.3, i=1, \ldots, n$, with a target expected return of $0.34 \%$. The results show that the multivariate ARMA-SCopMVN model has the highest average return of $4.81 \%$ with a higher risk of $3.20 \%$. Note that, model such as a-SCop-MVN has a higher average return of $4.62 \%$ and Sharpe ratio of 1.5803 with a much lower risk of $2.92 \%$.

Table 18 indicates that the allocation of assets to MSEXUK, S\&PCOMP, DAXINDX, AMSTEOE, HNGKNGI, BMUS10Y and BGILALL are different across models. It is also interesting to note that, only the model a-DCop-MVT short sell the asset TOKYOSE when the other models remain in long position. In particular, all model invested heavily in bond indices, i.e., BMUK10Y and BMBD10Y, ranging from $15 \%$ to $22 \%$, and $11 \%$ to $30 \%$, respectively.

\subsubsection{Efficient portfolio based on maximizing Sortino ratio without short sales}

Now, we consider the case of the efficient Sortino ratio portfolio without short sales. This portfolio is constructed by maximizing the Sortino ratio for a given risk-free rate with the same weight restrictions as in the previous models. Table 19 reports the performance results of the portfolios. The DCC-MVT model has the highest average return of 1.94\%, as well as the highest cumulative return and a Sharpe ratio. The other best models are aDCC-MVT, a-Dcop-MVT and SCop-MVT.

The highest asset allocation of this model mainly goes to bond indices, i.e., BMUK10Y, BMUS10Y, and BMBD10Y dominates the portfolio with about $7 \%$ to $20 \%$ of total investment. This is true for the entire model in mean-CVaR efficient portfolios without short sales. The average weights for the analyzed assets are presented in Table 20.

\subsubsection{Efficient portfolio based on maximizing Sortino ratio with short sales}

We construct an optimal portfolio based on maximizing Sortino ratio, with short sales such that the portfolio weight is restricted to be between $-0.3 \leq x_{i} \leq 0.3, i=1, \ldots, n$ at $95 \%$ probability level. Table 21 reports the performance measure for this optimization strategy. The results show that the DCC-MVT model has the highest monthly average return, cumulative return and Sharpe ratio. However, this model has the highest risk as compared to the other models. On the other hand, models like FDCC-MVN, aDCC-MVN, SCop-MVN also have a high return with a high Sharpe ratio at a much lower risk.

The average weights of asset portfolios constructed based on maximizing Sortino ratio are reported in Table 22. The asset allocation for assets MSEXUK, DAXINDX, AMSTEOE, HNGKNGI, and BGILALL are different across models. The allocation to TOKYOSE asset is high; around $10 \%$ to $25 \%$ of total investment in most of the models. It is interesting to note that only the ARMADCop-MVN model involves short sell for this optimization strategy.

\section{Conclusion}

This paper provides a comparative analysis of various multivariate DCC GARCH models using a portfolio allocation strategy as the loss function. We examined the portfolio's performance in terms of the returns, variance of returns, Sharpe ratio, and the value-atrisk. We applied different multivariate GARCH models, i.e., VAR, ARMA, DCC, aDCC, FDCC, GOGARCH and copula-GARCH models by using the normal and student distributions involving static and dynamic copulas. The use of the multivariate DCC-GARCH family is particularly appealing as it preserves the ease of estimation with a small number of parameters involved, thus solving the problems of dealing with a large number of parameters in other multivariate models. We considered different models for the asset allocation process and also for risk management purposes to see which is the best model in optimising the portfolio return. The analysis focuses on 12 assets consisting of four bonds and eight stock indices in the US, UK, Germany, Japan, Netherlands, Canada and Hong Kong. The results confirm the presence of heteroskedasticity, fat tails and volatility clustering in the asset returns of the data. We fitted the multivariate GARCH models to the portfolio returns to get the estimation of the variance-covariance matrix. Then, for the asset allocation, we considered the box-group constrained portfolio, whereby the weights for each asset and the weights of groups of selected assets are constrained by lower and upper bounds.

Specifically, for the minimum-variance portfolios, we found that the multivariate aDCC-MVT model outperformed the other models, by having the highest average monthly return, cumulative return and Sharpe ratio for both portfolios, with or without short sales. When we constructed the portfolio using the mean-variance optimization strategy such that we set the return of the portfolio to be greater than the target return, we found that the multivariate DCC-MVT, aDCC-MVN, VAR-MVN and VAR-SCop-MVN models have good performance measures as compared to the other models. As for maximizing the Sharpe ratio, the AR-GOGARCH-MVN and ARMA-MVN models outperformed the other models. For the mean-CVaR optimisation, we found that the models DCC-MVT, aDCCMVT, FDCC and a-DCop-MVT are comparatively the best models. Finally, when maximizing the Sortino ratio, we observed that once again the models DCC-MVT, aDCC-MVT, a-DCop-MVT and FDCC provide the best out-of-sample performance. Among the 
optimization strategies, the maximization of the Sortino ratio provides the best out-of-sample performance.

Two important results that emerge from this study is, first, the dynamic models are more capable of delivering better performance gains than the static models. These models reduce portfolio risk and improve the realized return in the out-of-sample period. Secondly, the results show that by adding copula functions to the models do not give a better performance when compared to the dynamic correlation models. While adding copula may capture additional characteristics in the data, it does not improve model selection outcomes in the performance of the portfolio. Our results are consistent with Weib (2013) and Ricetti (2013); the DCC models are not easily outperformed by any of the parametric copula models. That is, the copula model may not perform well when involving many related assets since we have only considered stock and bond indices in the portfolio. Overall, these findings are useful for practitioners who are involved in portfolio optimization and risk measurement in pension schemes. It will help the fund manager to decide the best investment strategies to ensure maximum benefit for its members.

This study can be extended in several ways. The problem has been solved using limited restrictions on the weights of the constructed portfolios. Future studies could incorporate different constraints to examine the robustness of the constructed portfolio and make a comparison. There are also other possible ways to extend the study by looking at different situations in which the copula model may perform better, i.e, by using other copula models, by looking at different asset allocation horizons, using multiple asset class in the portfolio, or considering other frequency of asset returns. Future studies could also consider incorporating exogenous factors-variables in the models, to see the effects it may have to the estimation of mean and variance-covariance matrix. It is interesting to note that, so far we have examined the asset management in a portfolio, the research could be extended by including the liabilities. In this context, the methodology proposed could be useful to pension managers and asset allocation purposes in pension schemes to ensure that enough assets are available to support pension liabilities. This study, therefore, can be a good benchmark for future related research.

\section{References}

Aielli, G. P. (2013). Dynamic conditional correlation: On properties and estimation. Journal of Business \& Economic Statistics, 31(3), $282-299$.

Alexander, C., \& Chibumba, A. (1996). Multivariate orthogonal factor GARCH. University of Sussex (Discussion Papers in Mathematics).

Alexander, S., Coleman, F., \& Li, Y. (2006). Minimizing CVaR and VaR for a portfolio of derivatives. Journal of Banking and Finance. 30, $583-605$.

Billio, M., Caporin, M., \& Gobbo, M. (2006). Flexible dynamic conditional correlation multivariate GARCH models for asset allocation. Applied Financial Economics Letters. 2, 123-130.

Bogentoft, E., Romeijn, H. E., \& Uryasev, S. (2001). Asset/liability management for pension funds using CVaR constraints. The Journal of Risk Finance, 3(1), 57-71. Bollerslev, T., Engle, R. F., \& Wooldridge, J. M. (1998). A capital asset pricing model with time varying covariances. Journal of Political Economy, 96, 116-131.

Boswijk, H., \& Weide, R.V.D. (2006). Wake me up before you GO-GARCH. Working paper, FEB: Amsterdam School of Economics Research Institute (ASE-RI).

Boubaker, H., \& Sghaier, N. (2013). Portfolio optimization in the presence of dependent financial returns with long memory: A copula based approach. Journal of Banking and Finance. 37(2), 361-377.

Broda, S. A., \& Paolella, M. S. (2009). A fast and accurate method for portfolio risk calculation. Journal of Financial Econometrics, 7(4), $412-436$.

Cappiello, L., Engle, R. F., \& Sheppard, K. (2006). Asymmetric dynamics in the correlations of global equity and bond returns. Journal of Financial Econometrics, 4(4), 537-572.

Chang, C. L., McAleer, M., \& Tansuchat, R. (2013). Conditional correlations and volatility spillovers between crude oil and stock index returns. The North American Journal of Economics and Finance, 25, 116-138.

Ding, Z. (1994). Time series analysis of speculative returns. San Diego: University of California (Ph.D. thesis).

Bouye, E., Durrleman, V., A.N.G.R., \& Roncalli, T. (2000). Copulas for finance - a reading guide and some applications. Techinal report, Credit Lyonnais.

Embrechts, P., McNeil, A., \& Straumann, D. (1999). Correlation and dependency in risk management: properties and pitfall. Working paper, ETH-Zentrum.

Engle, R. (2002). Dynamic conditional correlation: A simple class of multivariate generalized autoregressive conditional heteroskedasticity models. Journal of Business and Economic Statistics. 20, 339-350.

Engle, R., \& Kelly, B. (2012). Dynamic equicorrelation. Journal of Business and Economic Statistics. 30(2), $212-228$.

Engle, R. F., \& Colacito, R. (2006). Testing and valuing dynamic correlations for asset allocation. Journal of Business and Economic Statistics. 24(2), 238-253.

Engle, R. F., Lilien, D., \& Robins, R. (1987). Estimating time varying risk premia in the term structure: The ARCH-M model. Econometrice: Journal of the Econometric Society, 55(2), 391-407.

Ferstl, R., \& Weissensteiner, A. (2011). Asset-liability management under time-varying investment opportunities. Journal of Banking and Finance. 35, 182-192.

Hoevenaars, R. P., Molenaar, R. D., Schotman, P. C., \& Steenkamp, T. B. (2013). Strategic asset allocation with liabilities: Beyond stocks and bonds. Journal of Economic Dynamics and Control. 32, 2939-2970.

Huang, C. W., \& Hsu, C. P. (2015). Portfolio optimisation with GARCH-EVT-Copula-CVaR models. Banking and Finance Review, 7(1), $19-31$.

Hurlimann, W. (2014). On some properties of two vector-valued var and cte multivariate risk measures for archimedean copulas. ASTIN Bulletin: The Journal of the International Actuarial Association, 44(3), 613-633.

Joe, H. (1997). Multivariate Models and Multivariate Dependence Concepts, vol. 73. Chapman \& Hall.

Koivu, M., Pennanen, T., \& Koivu, A. R. (2005). Modeling assets and liabilities of a Finnish pension insurance company: A VEqC approach. Scandinavian Actuarial Journal. 1, 46-76.

Kresta, A. (2015). Application of garch-copula model in portfolio optimization. Financial Assets and Investing, 6(2), 7-20.

Kris Boudt, J. D., \& Laurent, S. (2013). Robust forecasting of dynamic conditional correlation garch models. Int. J. Forecast. $29(2), 244-257$.

Lee, M. C., Chiou, J. S., \& Lin, C. M. (2006). A study of value-at-risk on portfolio in stock return using DCC multivariate GARCH. Applied Financial Economics Letters, 2(3), 183-188.

Li, D. (2000). On default correlation: A copula function approach. Journal of Fixed Income, 9(4), 43-51.

Luo, C., Seco, L., \& Wu, L. L. B. (2015). Portfolio optimization in hedge funds by OGARCH and markov switching model. Omega, 57(Part A), 34-39.

Markowitz, H. (1952). Portfolio selection. The Journal of Finance, 7(1), 77-91.

Otranto, E. (2010). Asset allocation using flexible dynamic correlation models with regime switching. Quantitative Finance, 10(3), 325-338.

Patton, A. J. (2004). On the out-of-sample importance of skewness and asymmetric dependence for asset allocation. Journal of Financial Econometrics, 2(1), 130-168. Patton, A. J. (2006). Modelling asymmetric exchange rate dependence. International Economic Review, 47(2), 527-556.

Platanakis, E., \& Sutcliffe, C. (2014). Asset liability modelling and pension schemes: The application of robust optimization to USS. University of Reading (Discussion paper). Ricetti, L. (2013). A copula-garch model for macro asset allocation of a portfolio with commodities an out-of-sample analysis. Empirical Economics, 44, 1315-1336. 
Rockafellar, R. T., \& Uryasev, S. (2000). Optimization of conditional value-at-risk. Journal of Risk, 21-41.

Santos, A. A., \& Moura, G. V. (2014). Dynamic factor multivariate Garch model. Computational Statistics \& Data Analysis.

Sharpe, W. F., \& Tint, L. G. (1990). Liabilities-a new approach. Journal of Portfolio Management, 16(2), 5.

Silvennoinen, A., \& Terasvirta, T. (2015). Modeling conditional correlations of asset returns: A smooth transition approach. Econometrics Reviews, 34(1-2), 174-197. Sklar, A. (1959). Fonctions de rTpartition a n dimensions et leurs marges. Publications de l'Institut de Statistique de L'UniversitT de Paris, 8, $229-231$.

Tsay, R. S. (2013). An introduction to analysis of financial data with R. John Wiley \& Sons.

Weib, G. N. F. (2013). Copula-garch versus dynamic conditional correlation: An empirical study on var and es forecasting accuracy. Review of Quantitative Finance and Accounting, 41(2), 179-202.

Weide, R. V. D. (2002). GO-GARCH a multivariate generalized orthogonal GARCH model. Journal of Applied Econometrics, 17, $549-564$.

Wu, C. C., \& Lin, Z. Y. (2014). An economic evaluation of stock-bond return comovements with copula based GARCH models. Quantitative Finance, 14(7), 1283-1296.

Zhang, K., \& Chan, L. W. (2009). Efficient factor GARCH models and factor DCC models. Quantitative Finance(1). 